\title{
LA-UR-15-27608
}

Approved for public release; distribution is unlimited.

Title: $\quad$ Using xRage to Model Heat Flow for Experiments to Measure Opacities in HED Plasmas

Author(s): $\quad$ Elgin, Laura E.

Intended for: $\quad$ Student presentation of summer research at her home university.

Issued: $\quad$ 2015-09-30 
Disclaimer:

Los Alamos National Laboratory, an affirmative action/equal opportunity employer,is operated by the Los Alamos National Security, LLC for the National NuclearSecurity Administration of the U.S. Department of Energy under contract DE-AC52-06NA25396. By approving this article, the publisher recognizes that the U.S. Government retains nonexclusive, royalty-free license to publish or reproduce the published form of this contribution, or to allow others to do so, for U.S. Government purposes. Los Alamos National Laboratory requests that the publisher identify this article as work performed under the auspices of the U.S. Departmentof Energy. Los Alamos National Laboratory strongly supports academic freedom and a researcher's right to publish; as an institution, however, the Laboratory does not endorse the viewpoint of a publication or guarantee its technical correctness. 


\section{Using xRage to Model Heat Flow for Experiments to Measure Opacities in HED Plasmas}

\section{Laura Elgin}

\section{September 15, 2015}

U.S. Department of Energy, through the NNSA-DS and SC-OFES Joint Program in High-Energy-Density Laboratory Plasmas, grant number DE-NA0001840. 


\section{Outline}

- Introduction \& Motivation

- Pleiades Platform at NIF

- xRage Simulation Overview

- Simulation Data

- Comparison with Previous Simulation Results

- Analysis

- Conclusions \& Future Work

- Acknowledgements \& References 


\section{Solar Abundance Problem}

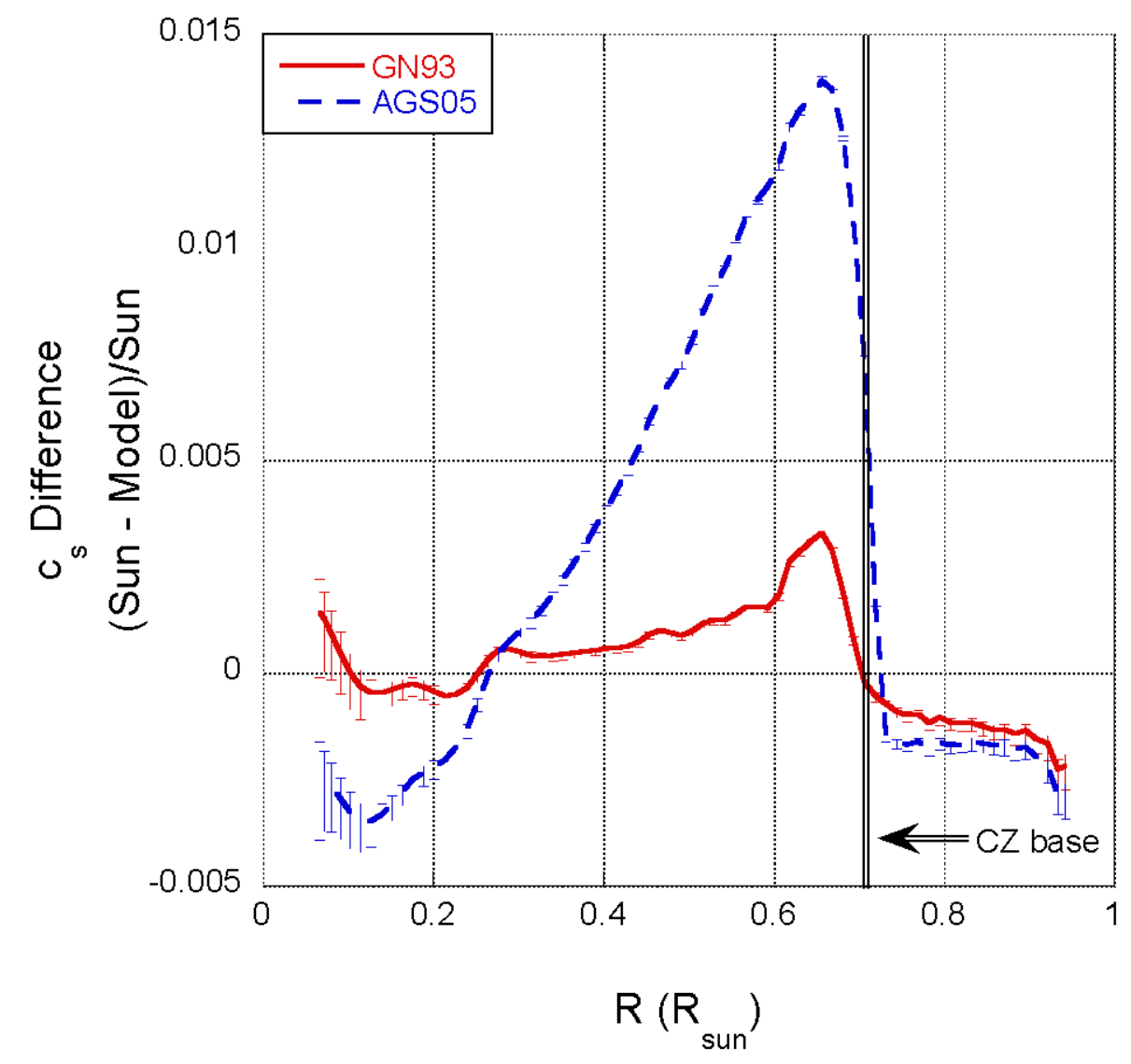

- Recent solar models find significantly lower abundances of $\mathrm{C}, \mathrm{N}$, and $\mathrm{O}$ than previous models.

- The difference in the sound speed between the models and the sun more than tripled beneath the convection zone.

- Solar models using the updated abundances do not agree with measured data from neutrinos and helioseismology.

- Small differences in sound speed correspond to large revisions to elemental abundances, opacities, or other properties of matter in the solar interior. 


\section{Impact of Opacities on Solar Abundances}

- Possible explanations for solar abundance discrepancy include:

$-\mathrm{A}$

$-B$

$-\mathrm{C}$

- Ad-hoc revisions to opacities of [list elements \& \% increase] yield solar abundances in agreement with [helioseismic and \&\&] measurements; however, there is no physical justification.

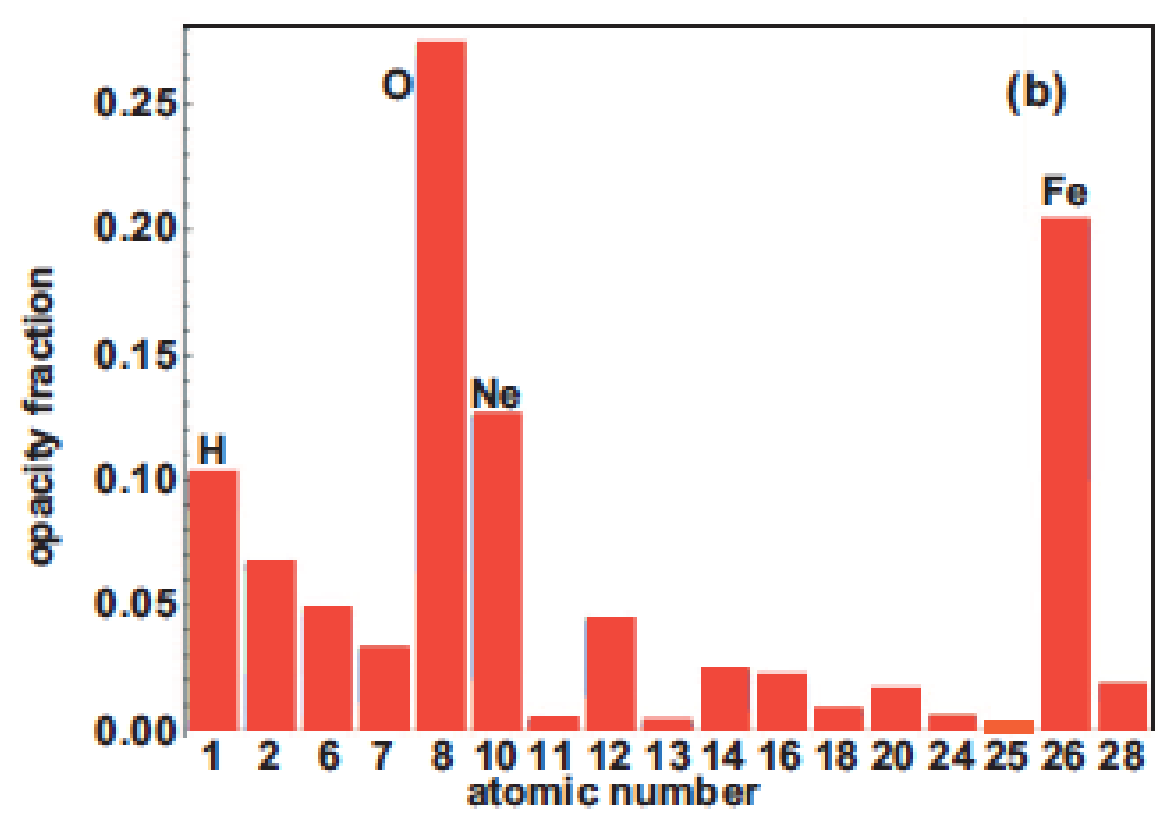

The histogram is the fractional contribution to the Rosseland mean evaluated near the base of the solar convection zone using the OPAS opacity model. (Bailey 2009) 


\section{Opacity Models Do Not Agree}

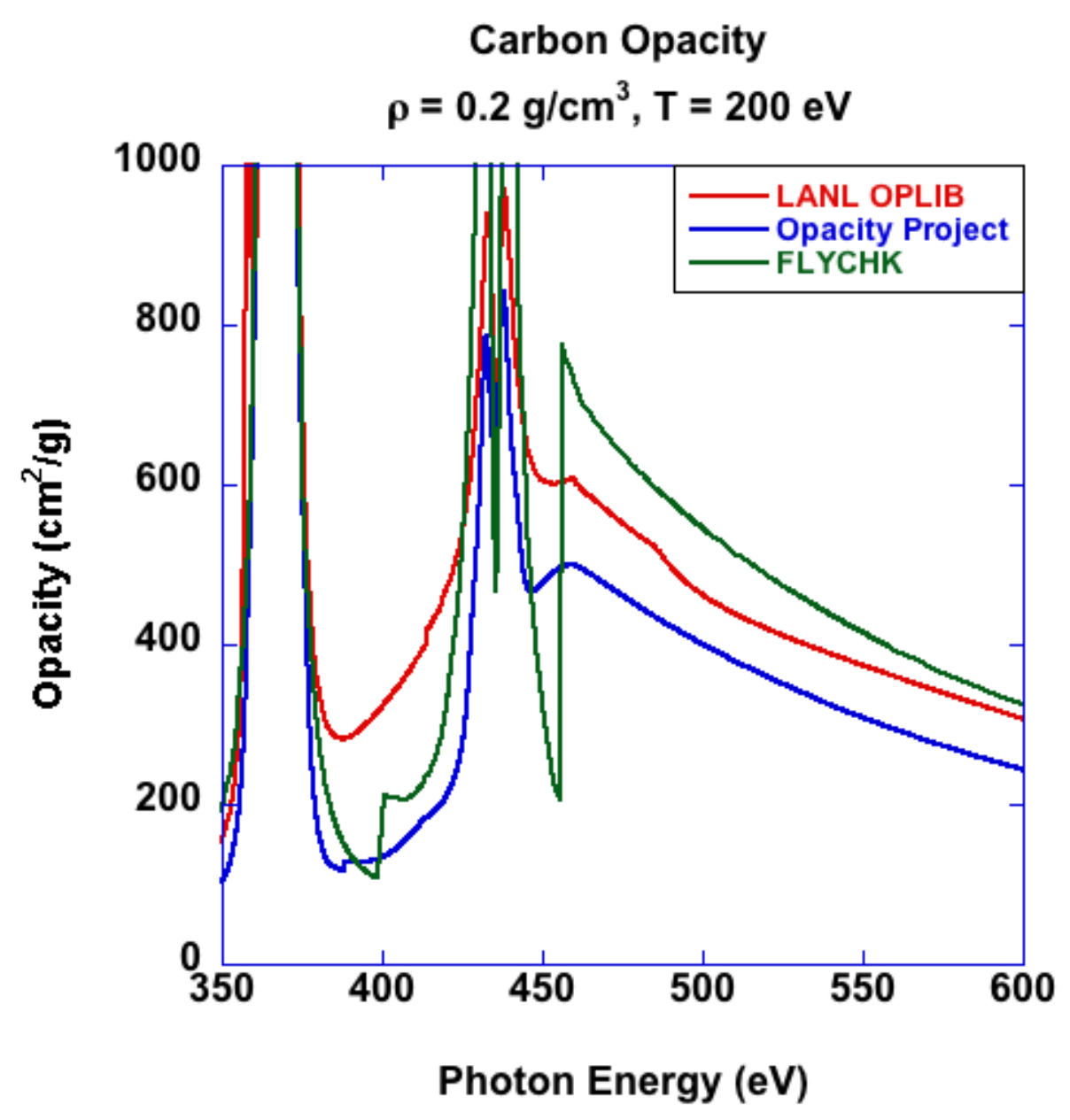




\section{NIF Can Achieve Conditions Relevant to BCZ}

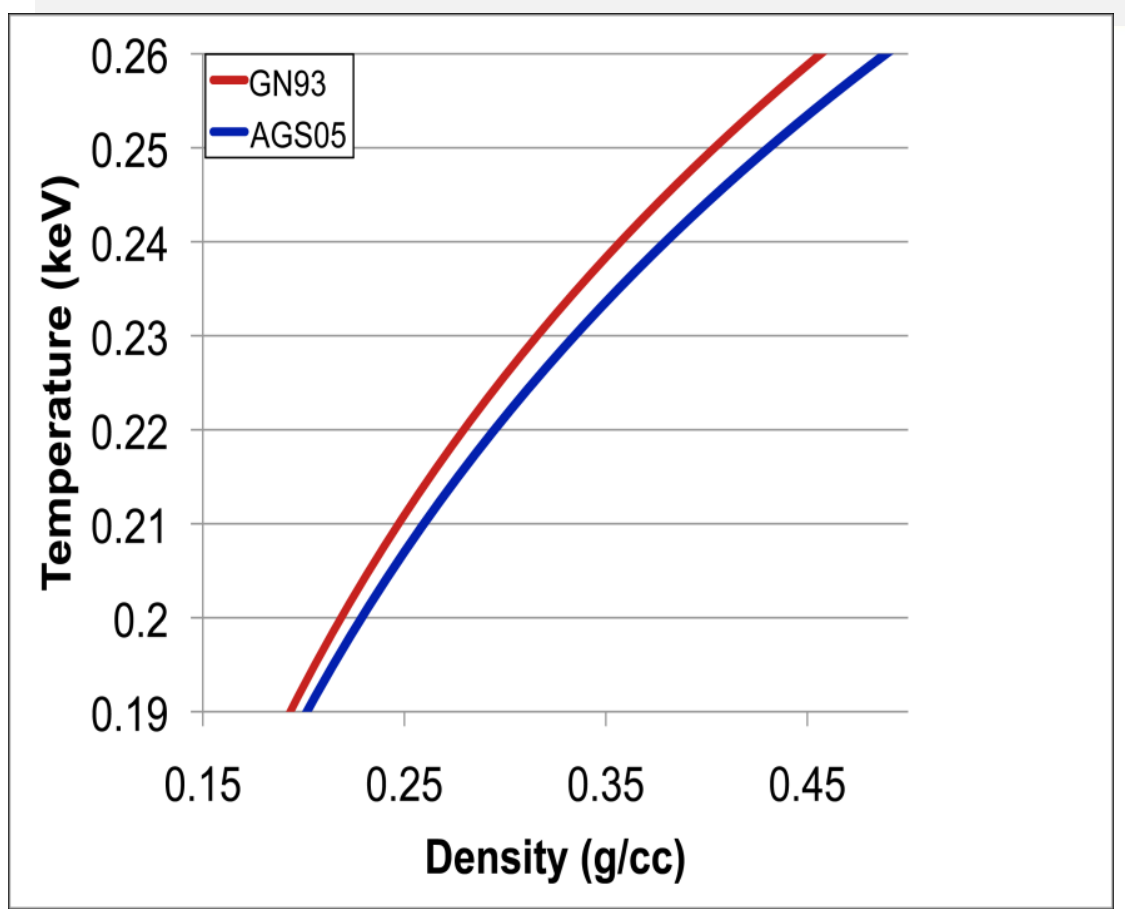

Temperature and density from $\mathrm{r} 0.6 \mathrm{R}$ (on the right end) to $0.7 \mathrm{R}$ (on the left end), which

corresponds to just below the base of the $\mathrm{CZ}$, which is at $0.71 \mathrm{R}$ in the GN93-based model and $0.73 \mathrm{R}$ in the AGS05-based model.

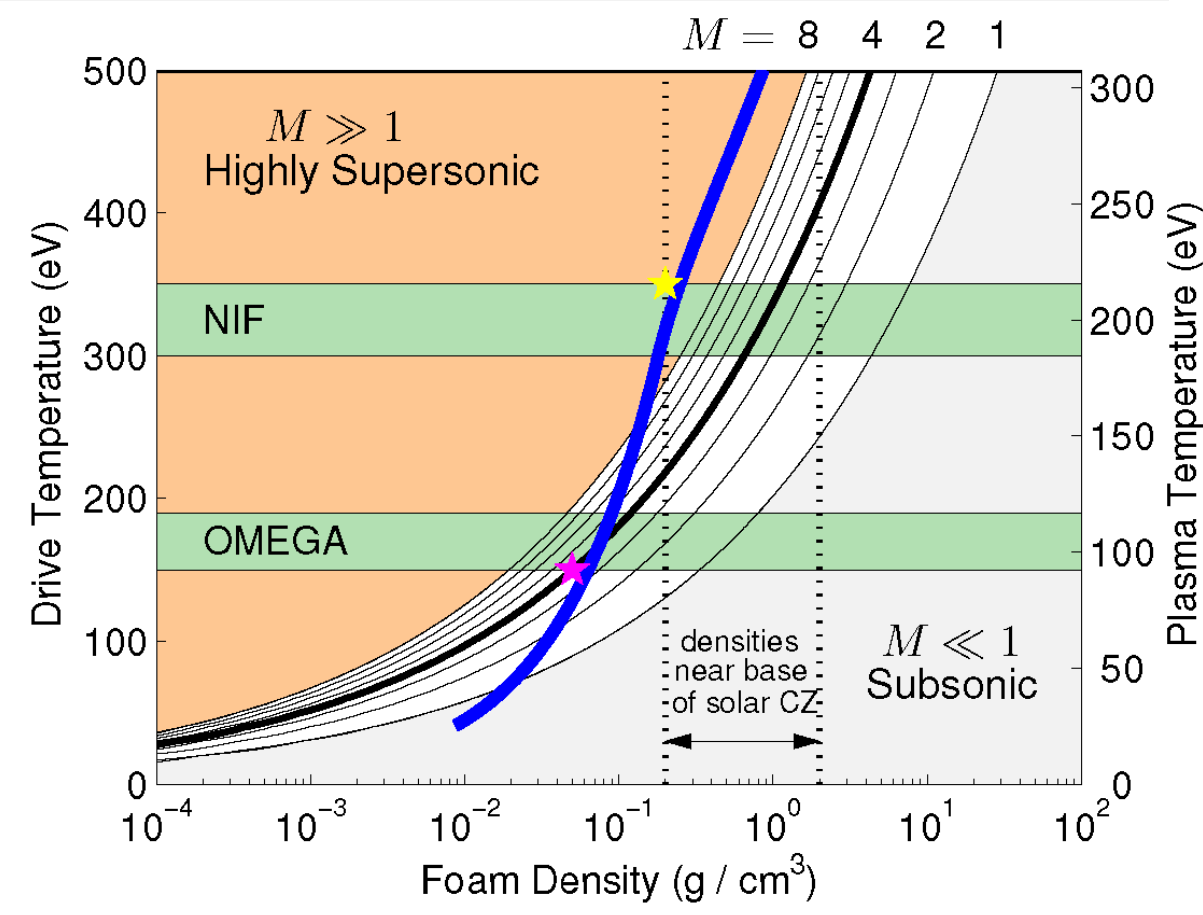

Fig. from Chris Orban

\section{LosAlamos}




\section{Proposed Experiment \& Aims}

- Measure opacities of $\mathrm{C}, \mathrm{O}, \mathrm{N}$ at temperatures \& densities corresponding to base of solar convection zone.

- Compare data with existing opacity models; validate models or inform development of new models

- Will use spectrometer to measure "flat" parts of opacity spectra (not resolve peaks)

- Use measured opacities in solar evolution models and see if this yields abundances consistent with helioseismology data

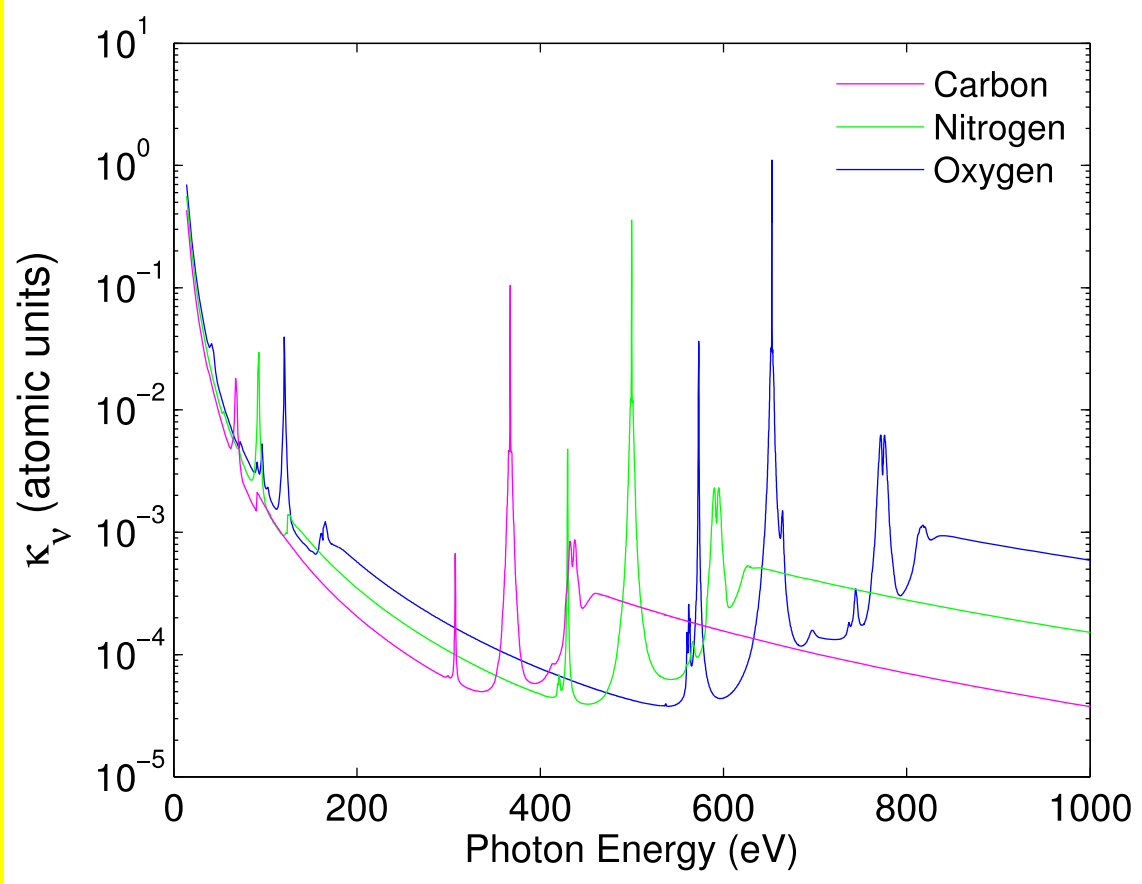

From OP model Reference?

Temperature for plot above?? 


\section{Pleiades Platform}

(a)

\section{Au M-band}

absorbing SiO2

aerogel Disc

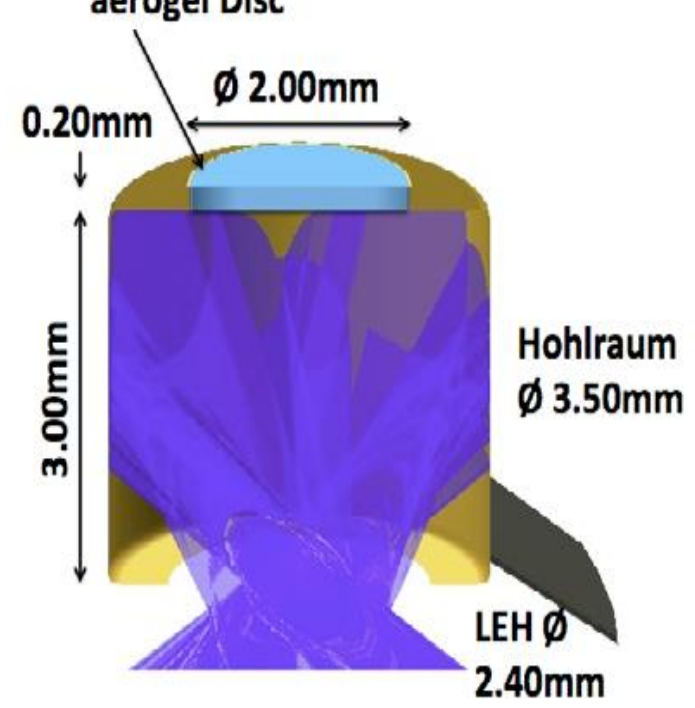

Moore et al 2013.
- Laser-driven half-hohlraum emits X-rays that drive supersonic radiation wave in foam targets.

- Previous experiments at NIF [ref] have demonstrated a hightemperature (>340 eV) X-ray radiation source with a quasiPlanckian distribution.

- Radiative transfer in foam target approximated by analytic solution for Marshak wave (constant temperature source driving a diffusive radiation wave). (b)

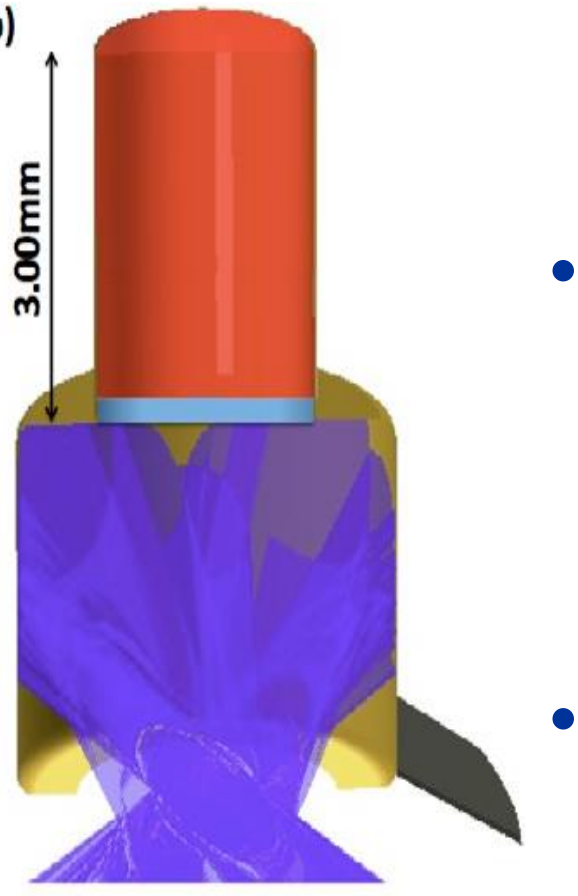




\section{Pleiades Platform \& Opacity Measurements}

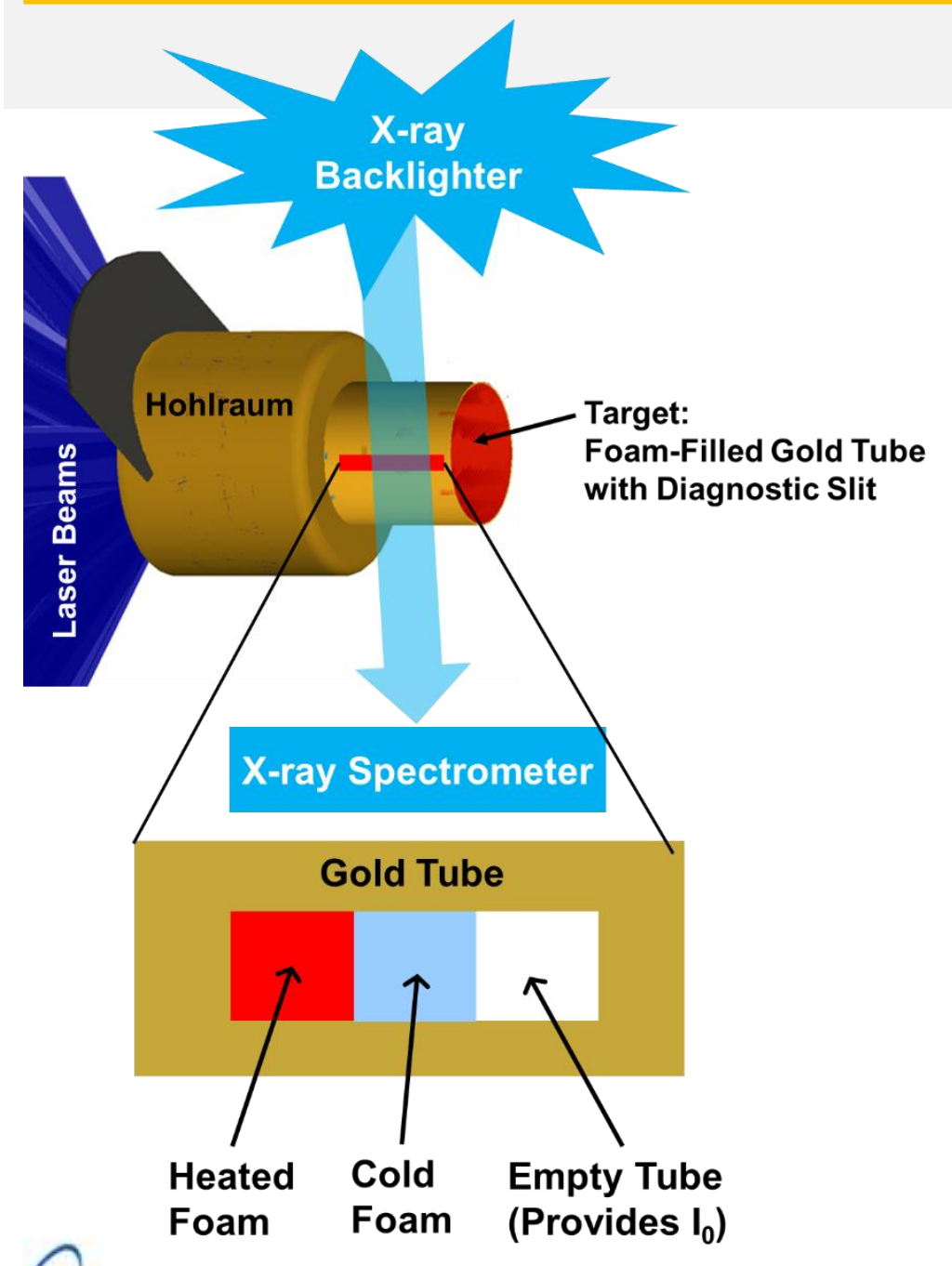

- Density of cold foam measured beforehand.

- X-ray source from hohlraum creates highly supersonic radiation wave that heats foam target.

- Density of heated foam remains constant and equal to density of cold foam over a time scale of a couple ns, before hydrodynamic effects create temperature and density gradients.

- During this brief time window, opacities of both the hot and cold material are measured. 


\section{Target Used in Simulation}

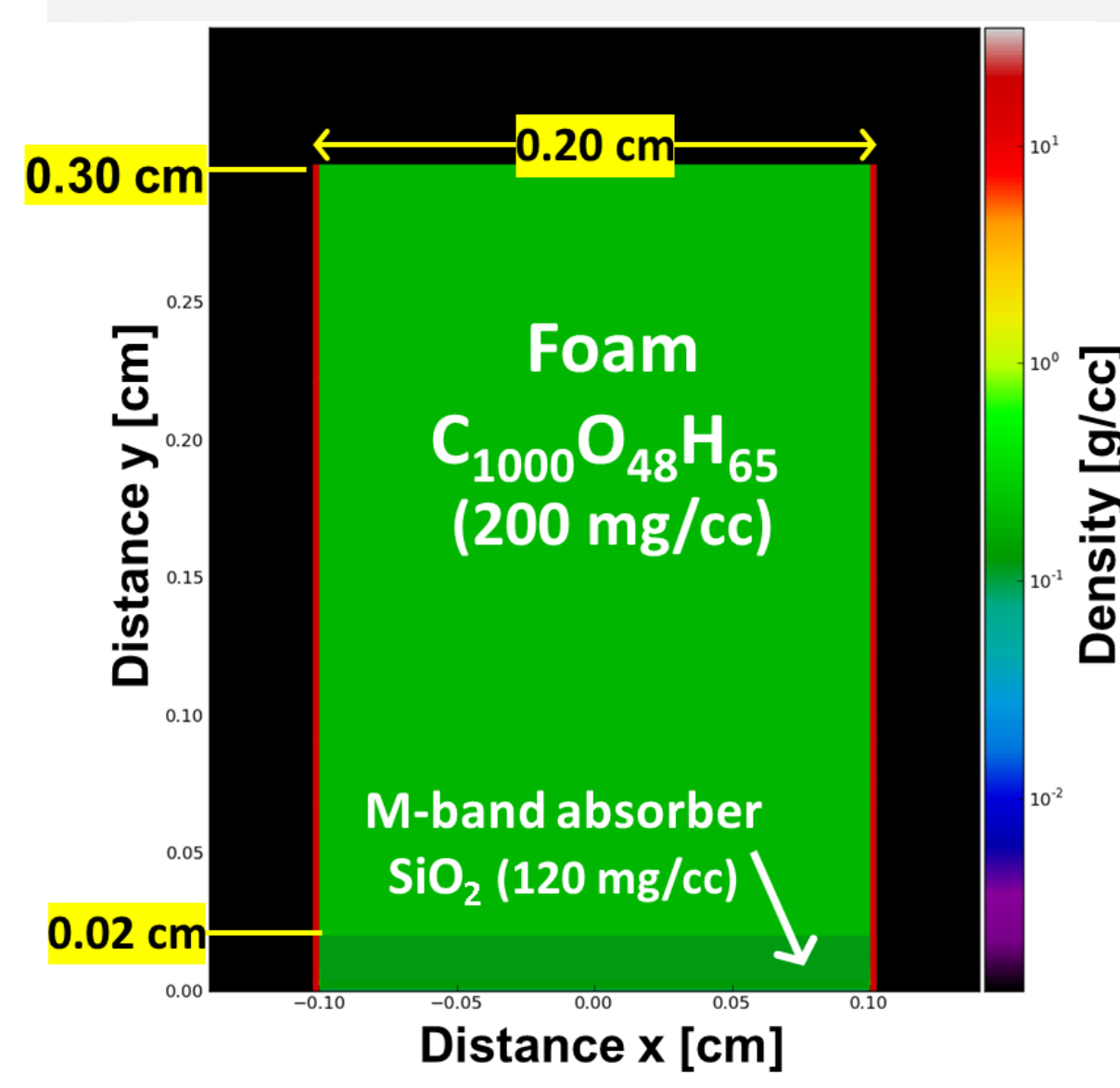

- 200 mg/cc Carbon foam.

- $\mathrm{SiO}_{2}$ disk absorbs M-band radiation emitted from hohlraum.

- Gold tube with $25 \mu \mathrm{m}$ wall thickness serves as a tamper.

- No diagnostic slots in tube (cylindrically-symmetric geometry enables 2D simulation). 


\section{Hohlraum Emission \& X-Ray Source}

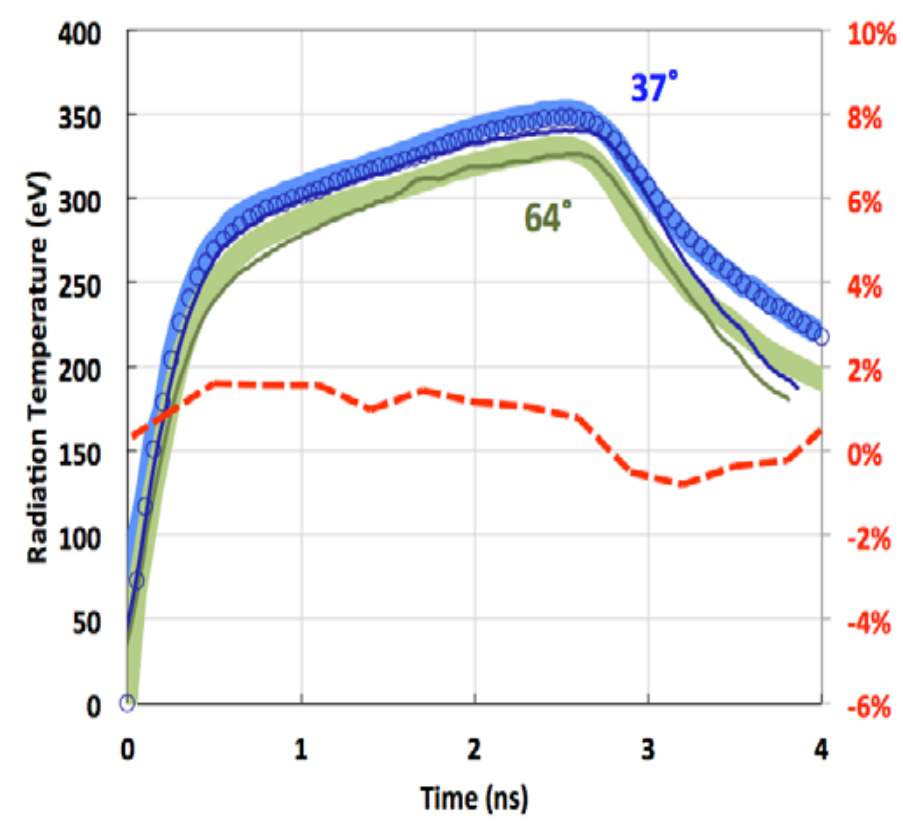

Fig. 3. Measurement of the hohlraum temperature through the LEH shown for two separate shots at 37 deg (dark-gray line and open circles) and 64 deg (light-gray line). Line widths are representative of the error bar in the measurement. NYM simulations of the emission are shown as solid black

lines. The percentage difference between two shots is shown on the right axis (dotted gray line).

Moore 2013.

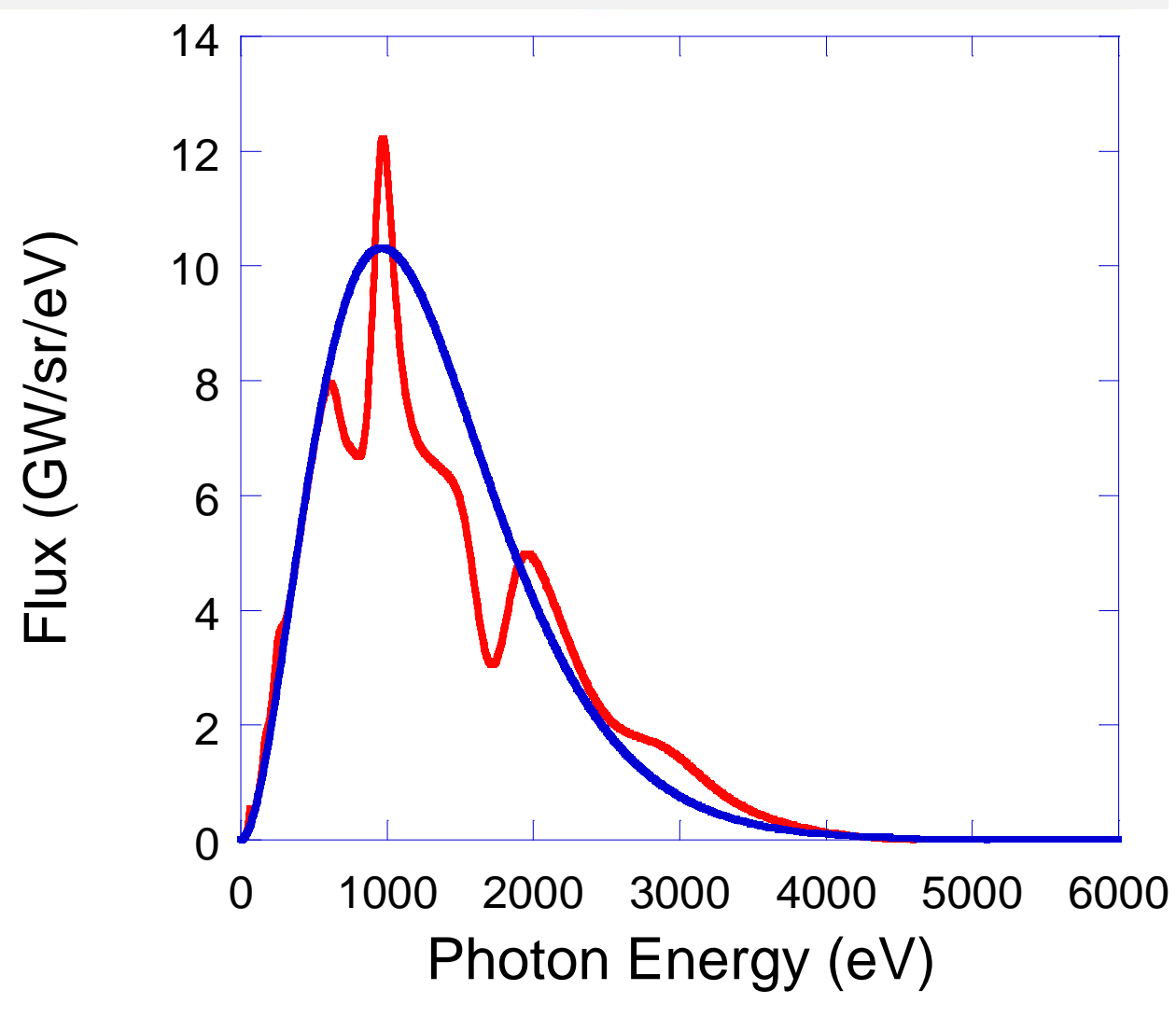

Katie Mussack

\section{Los Alamos}

NATIONAL LABORATORY

$$
\text { (ST, } 1943
$$




\section{xRage Simulation: Physical Model \& Inputs}

- "Cut-off" problem:

- Hohlraum not included

- 350 eV Planckian temperature source applied at target boundary

- Multi-group diffusion model, 2T NLTE

- Opacities \& EOS from LANL [unclassified] Sesame tables
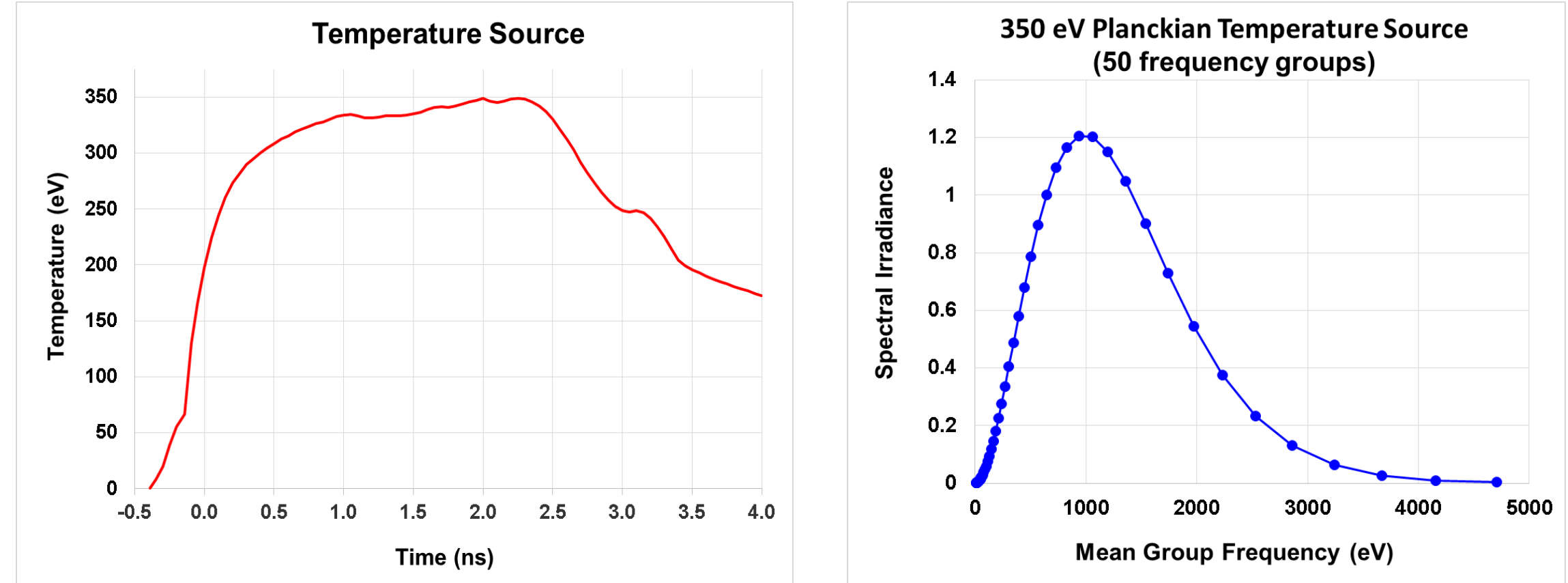


\section{xRage Simulation: Data Output}

- 2D data measured every 1 ps:

- Density (rho)

- Material Temperature (tev)

- Radiation Temperature (rev)

- Pressure (prs)

- Sound Speed (snd)

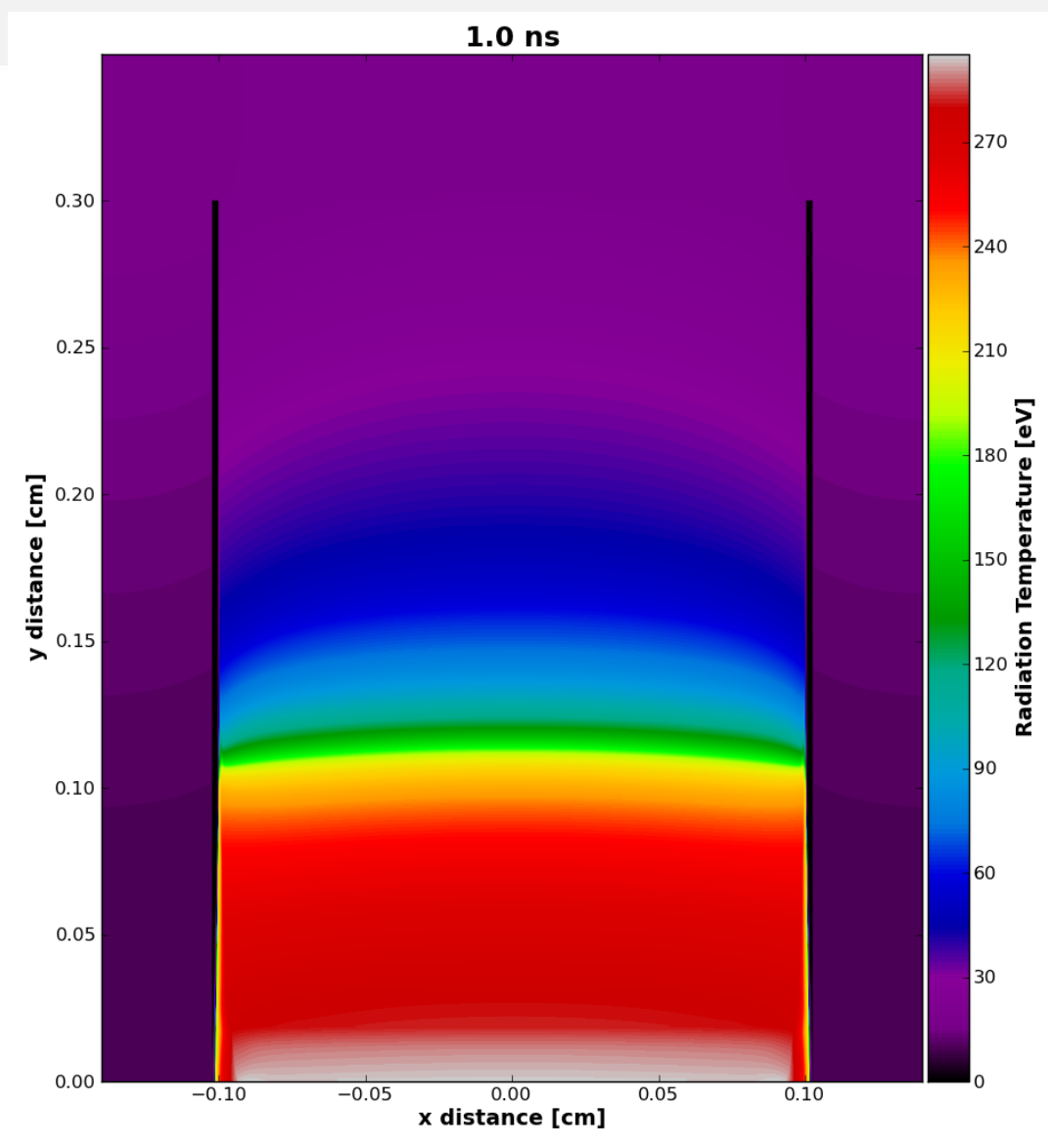




\section{Radiation Front Location}

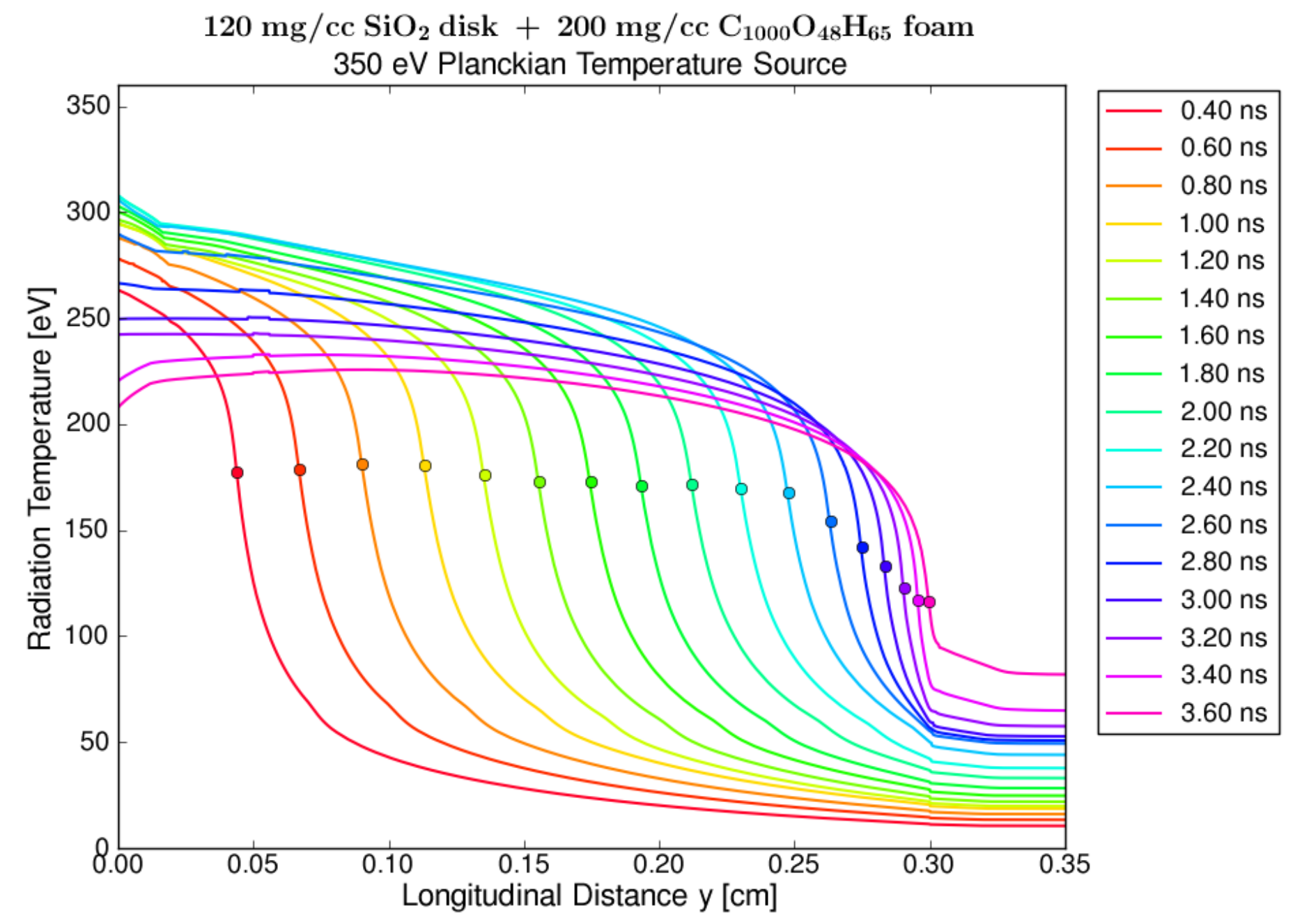

\section{- Los Alamos}

NATIONAL LABORATORY 


\section{Radiation Front Mach Number}

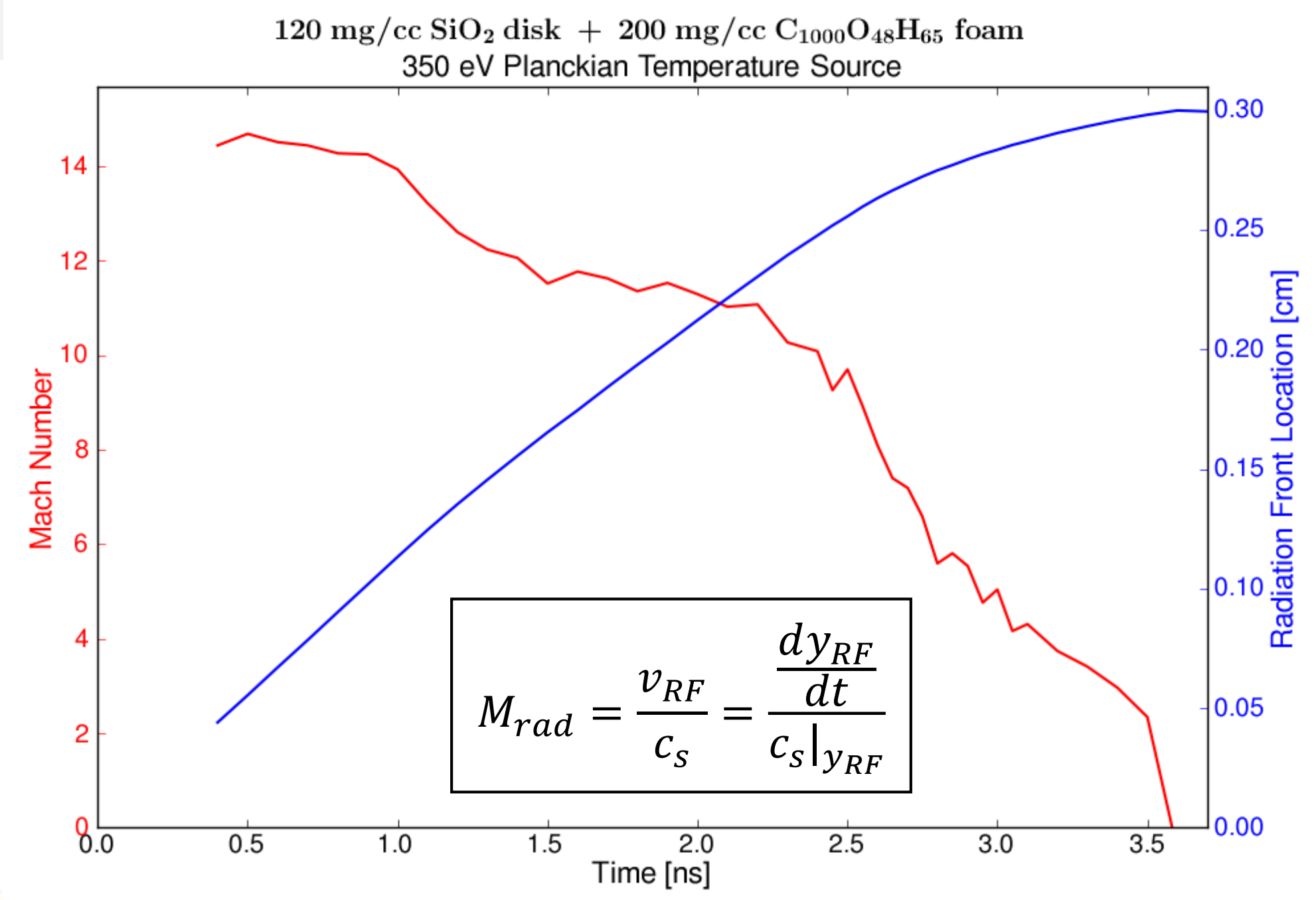

\section{Los Alamos}




\section{Material Temperature at $\mathrm{BCZ}$}

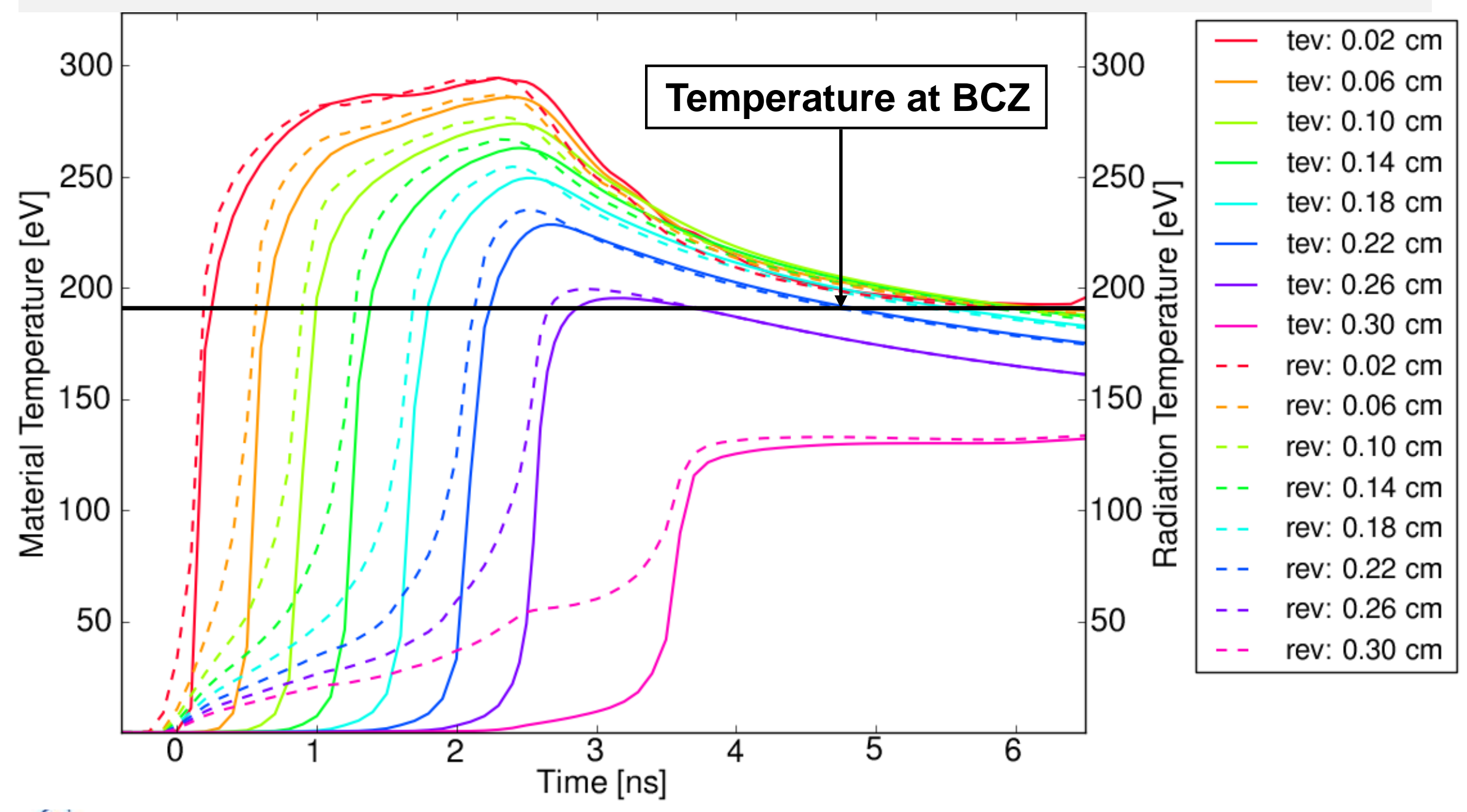




\section{Comparison with Previous Results}

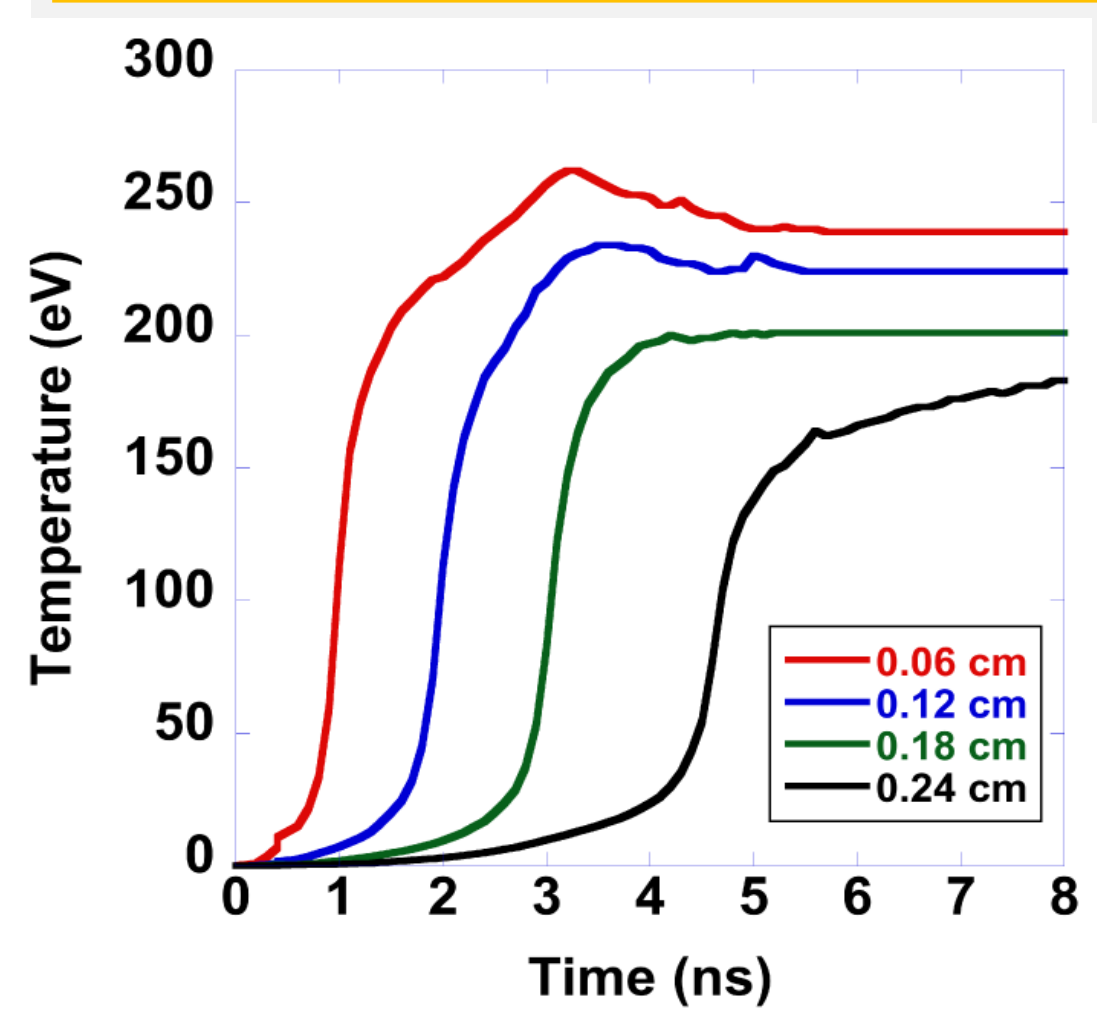

- 200 mg/cc Carbon target

- Implicit Monte Carlo (IMC) Simulation

- 320 eV Frequency-Dependent Source

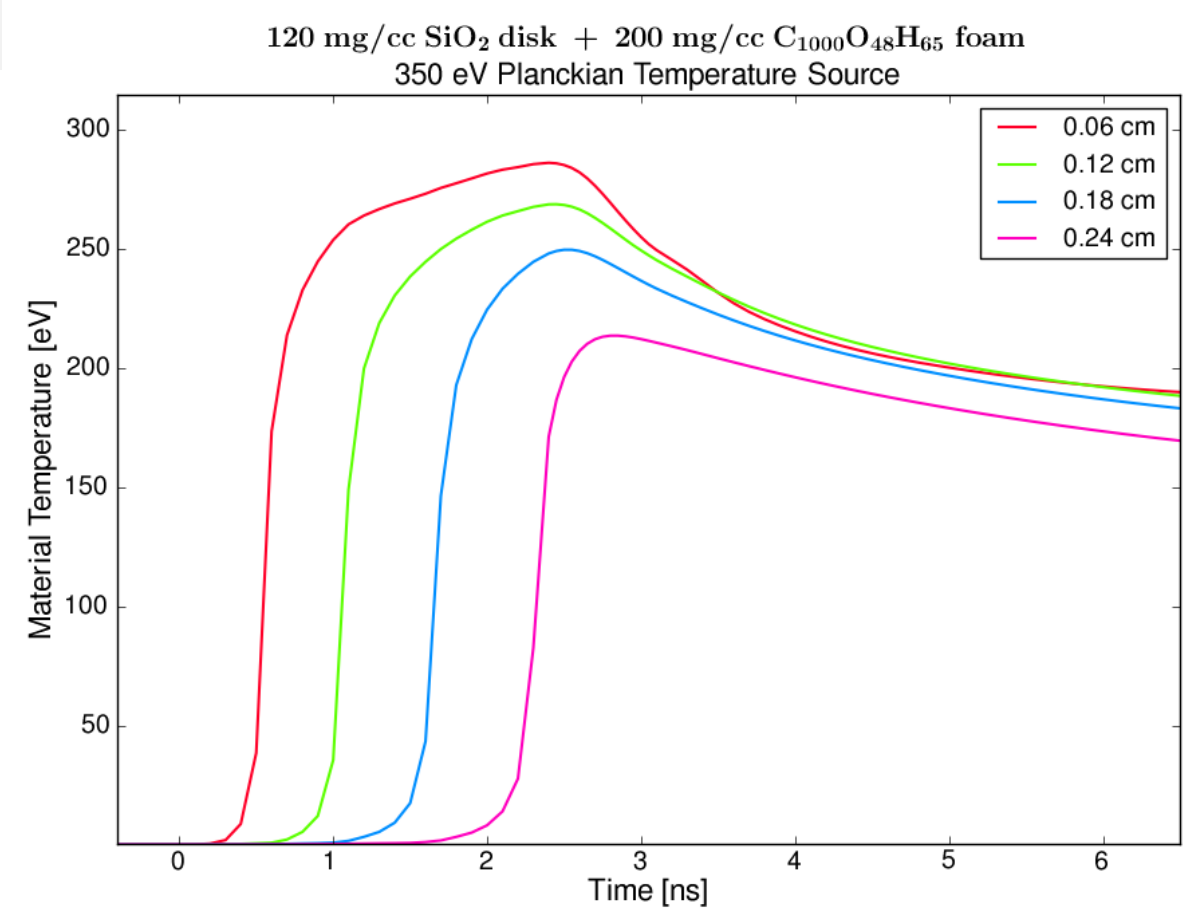

- 200 mg/cc Carbon target with $\mathrm{SiO}_{2}$ disk

- Multi-Group Diffusion Simulation

- 350 eV Planckian Temperature Source 


\section{Frequency-Dependent Source vs. Planckian}
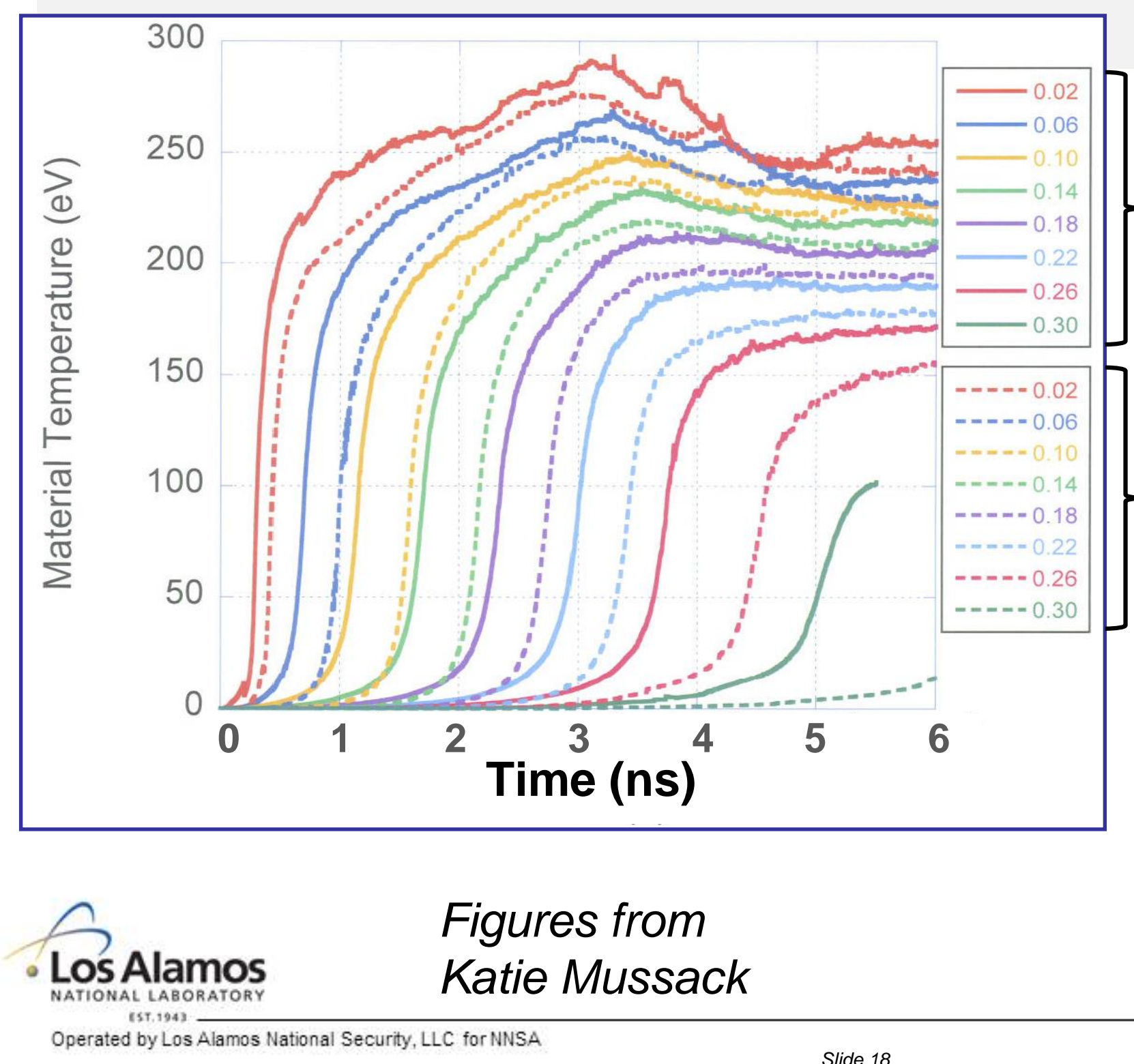

Figures from

Katie Mussack

\section{FDS}

Planckian

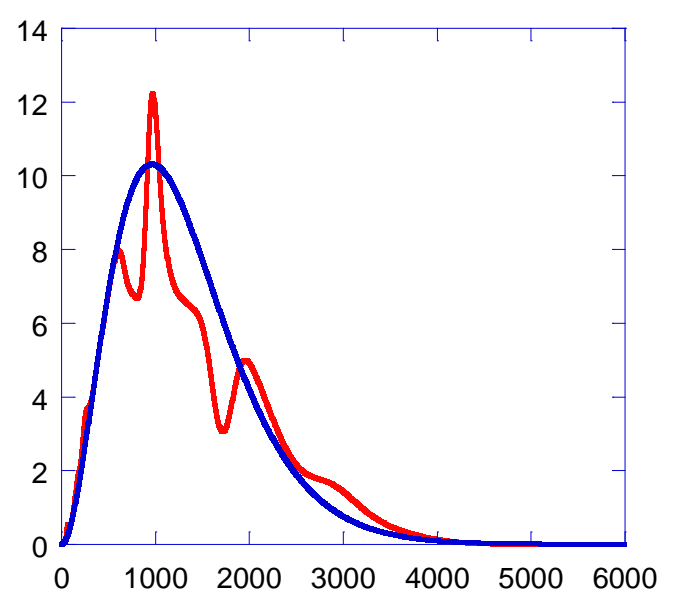




\section{Implicit Monte Carlo vs. Multi-Group Diffusion}

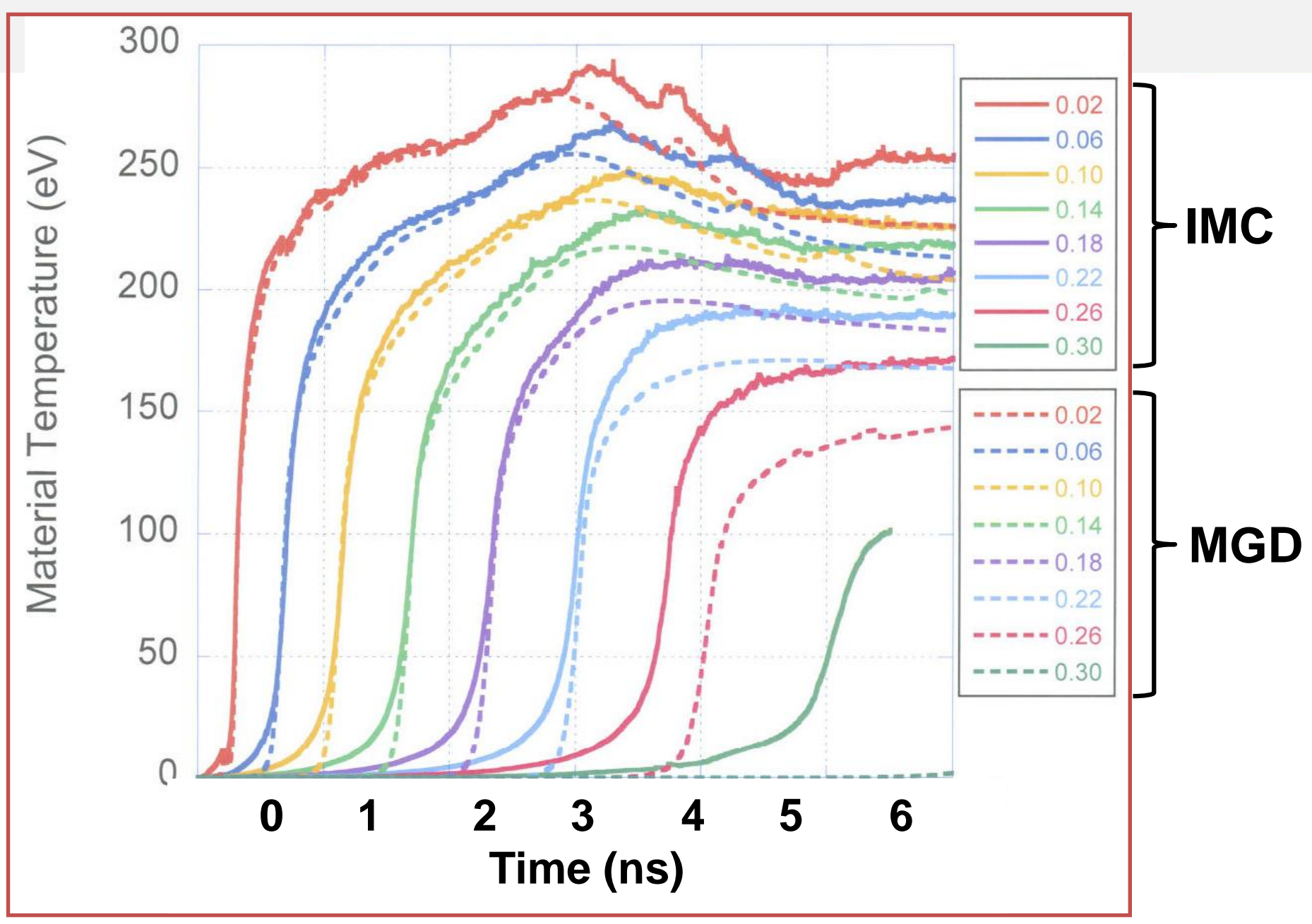

- LosAlamos For $\mathbf{t}<\mathbf{2 . 5}$ ns, not much difference between IMC \& MGD. NATIONAL LABORATORY 


\section{Diffusion Approximation}

- Analytic solution (1D, Cartesian) for supersonic diffusive radiation wave with constant temperature drive:

$$
\begin{aligned}
& \left(\frac{T}{T}\right)^{-n} \quad \text { Diffusive Limit (optically thick): } \\
& \kappa=\kappa_{0}\left(\frac{T}{T_{0}}\right) \\
& \varepsilon=\varepsilon_{0}\left(\frac{T}{T_{0}}\right)^{m} \\
& y_{R F}=\sqrt{\frac{32}{3\left(n+4-\frac{3}{2} m\right)} \frac{t \sigma T^{4}}{\kappa \rho^{2} \varepsilon}}
\end{aligned}
$$

Free-Streaming Limit (optically thin):

$$
y_{R F}=\frac{t \sigma T^{4}}{\rho \varepsilon}
$$




\section{Temperature Source Function \& Opacity}

Diffusive Limit (optically thick):

$y_{R F}=\sqrt{\frac{32}{3\left(n+4-\frac{3}{2} m\right)} \frac{t \sigma T^{4}}{\kappa \rho^{2} \varepsilon}}$

Rosseland Mean Opacity:

$$
\kappa_{R}(T)=\frac{\int_{0}^{\infty} \kappa_{v} \frac{d I}{d T} d E_{v}}{\int_{0}^{\infty} \frac{d I}{d T} d E_{v}}
$$

Free-Streaming Limit (optically thin):

$$
y_{R F}=\frac{t \sigma T^{4}}{\rho \varepsilon}
$$

Planckian Temperature Source:

$$
I\left(E_{v}, T\right)=\frac{2 e^{4} E_{v}{ }^{3}}{h^{3} c^{2}} \frac{1}{\exp \left(\frac{E_{v}}{T}\right)-1}
$$




\section{Limits of MGD Model \& Temperature Source}

- $y_{R F} \sim t$

- Free-streaming radiation

- Target material optically thin

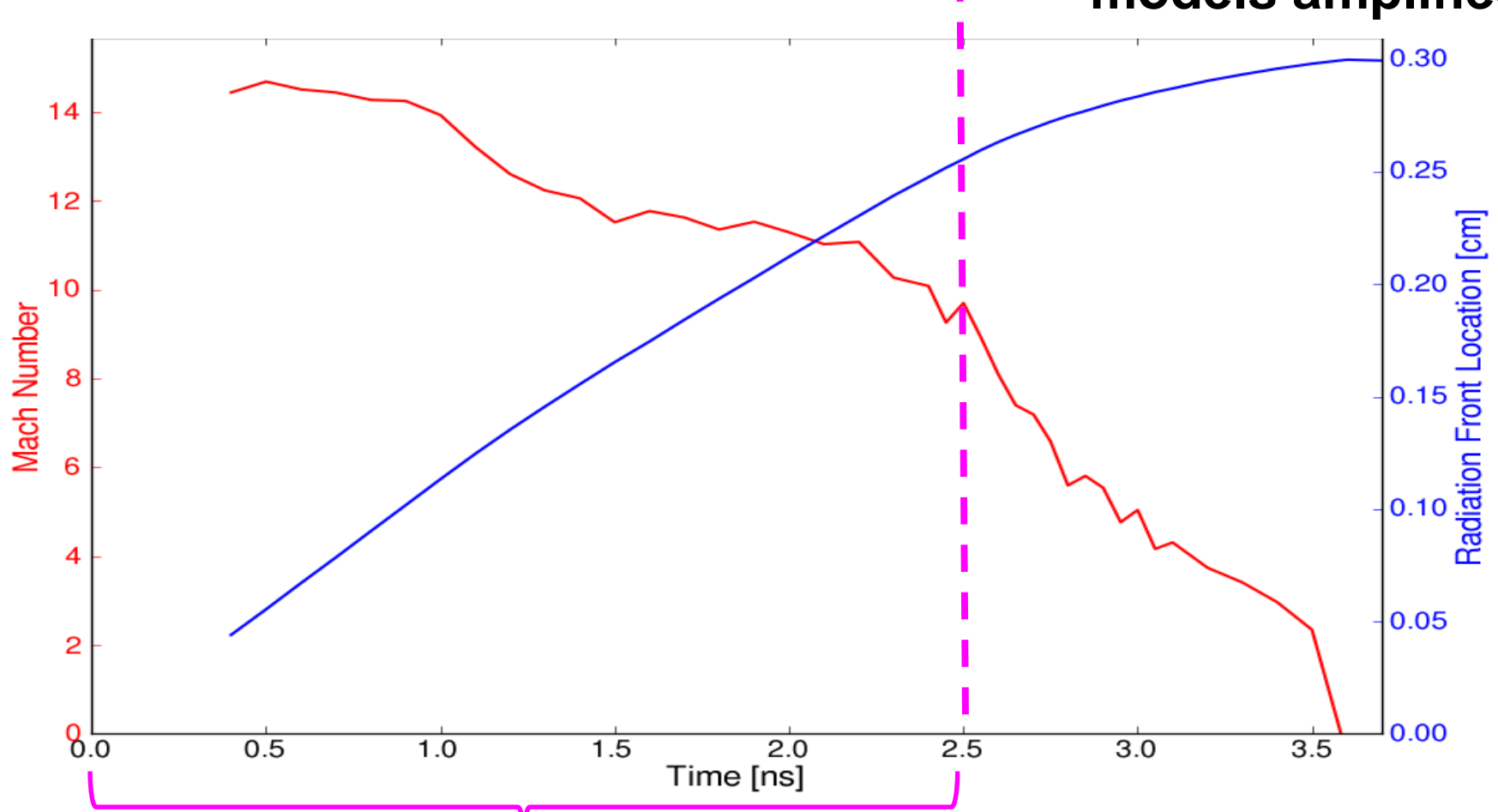

- Neither optically thin nor thick

- $T(t)$ rapidly decreasing

- Diffusive model less accurate

- Differences in temperature source models amplified 


\section{Issues with Carbon Target}

- Temperature source \& target composition in this simulation did not achieve desired conditions at base of solar convection zone.

- Absorption spectrum of Carbon:

- NIF does not currently have spectrometer for $<600 \mathrm{eV}$

- If hot target is transparent to X-ray spectrum from Hohlraum, it will likely be transparent to back-lighter source used for absorption spectroscopy (target may be too thin and too transparent to measure opacity)

- Gold tube ablation 


\section{Oxygen-Doped Targets}
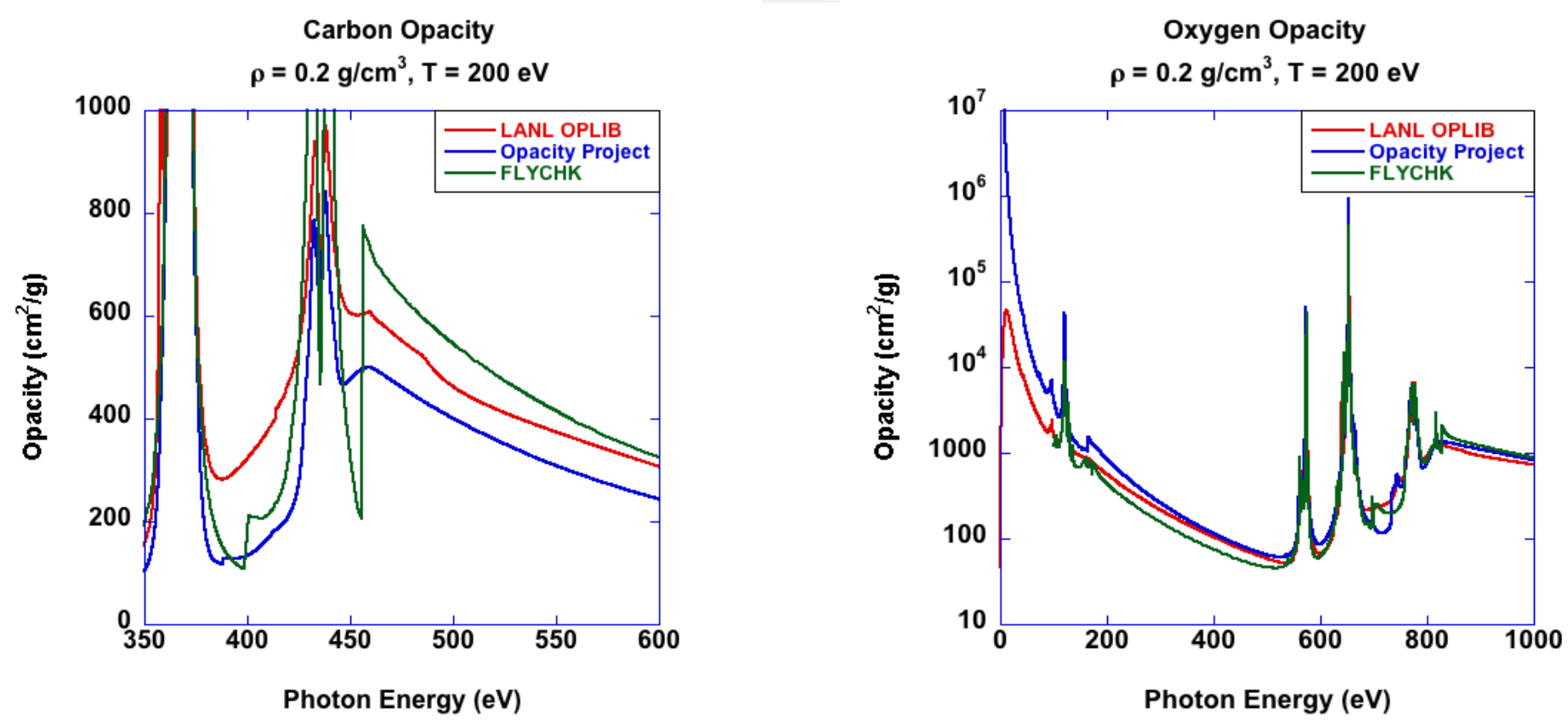

- New simulation for oxygen-doped target.

- More detailed analysis of radiation drive temperature, material temperature and density, and opacity. 


\section{Gold Tube Ablation}

\section{4 ns}

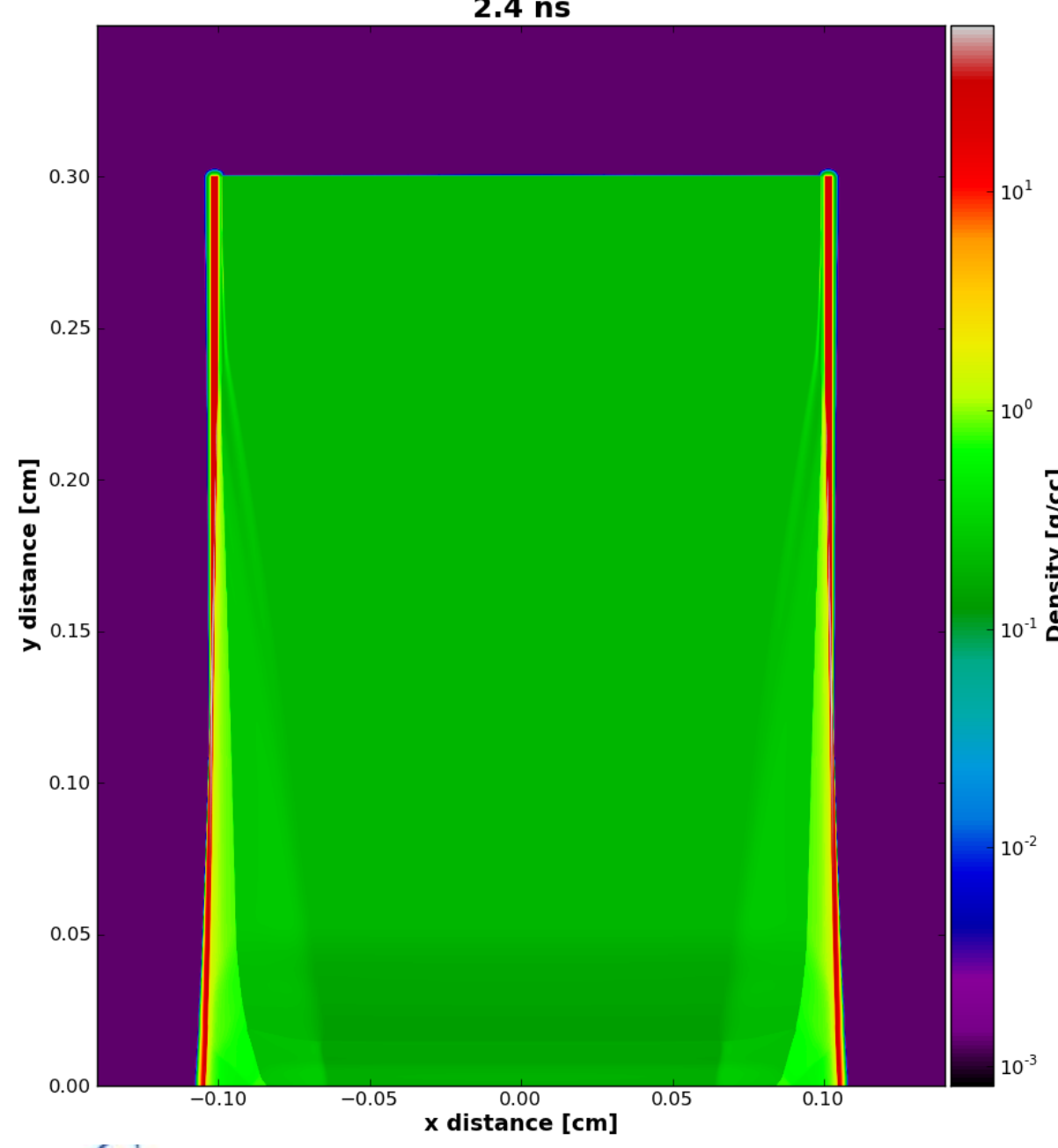

$2.4 \mathrm{~ns}$

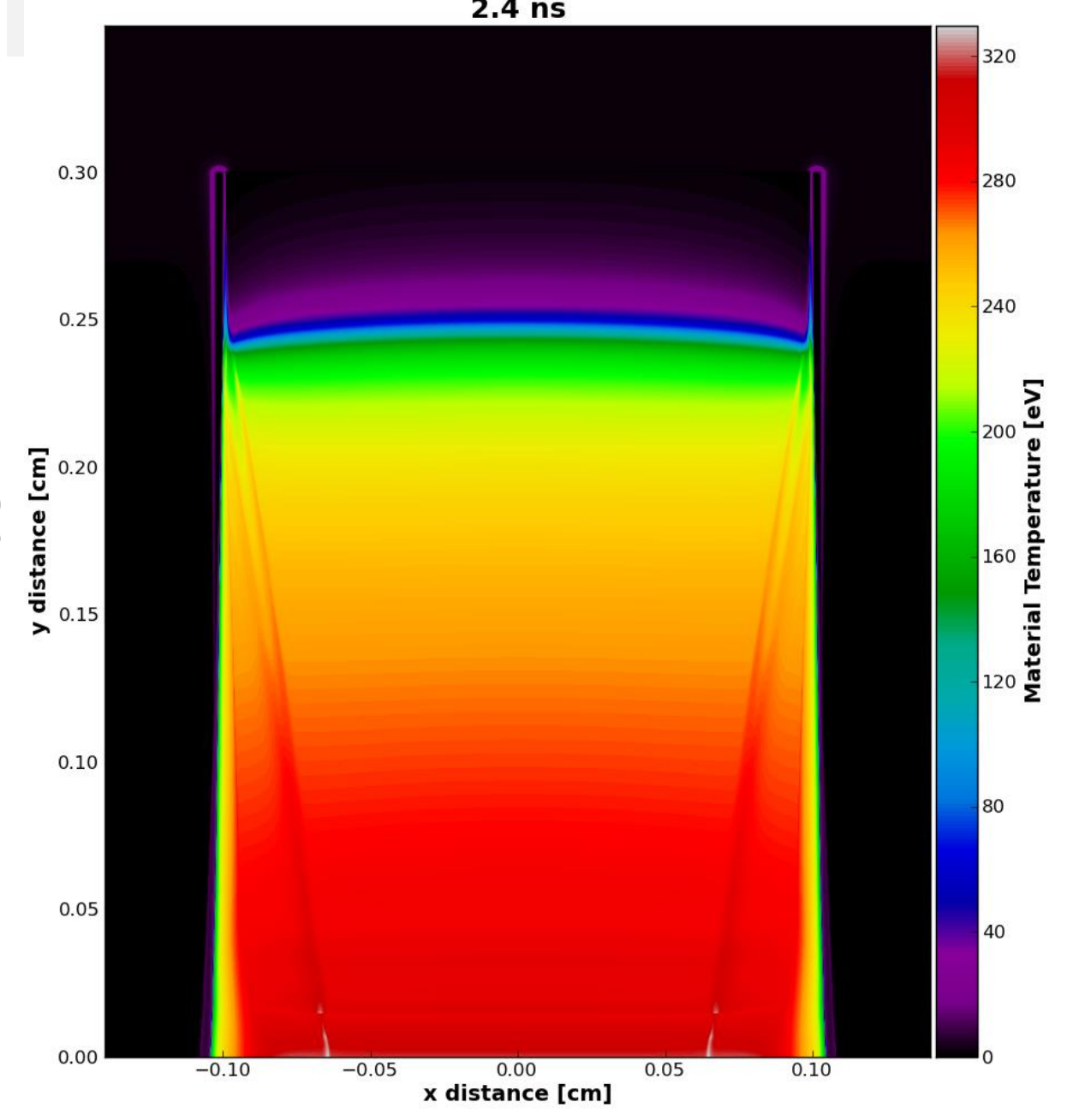

- Los Alamos

NATIONAL LABORA
[ST, 1943

Operated by Los Alamos National Security, LLC for NNSA 


\section{Temperature and Density Profiles}
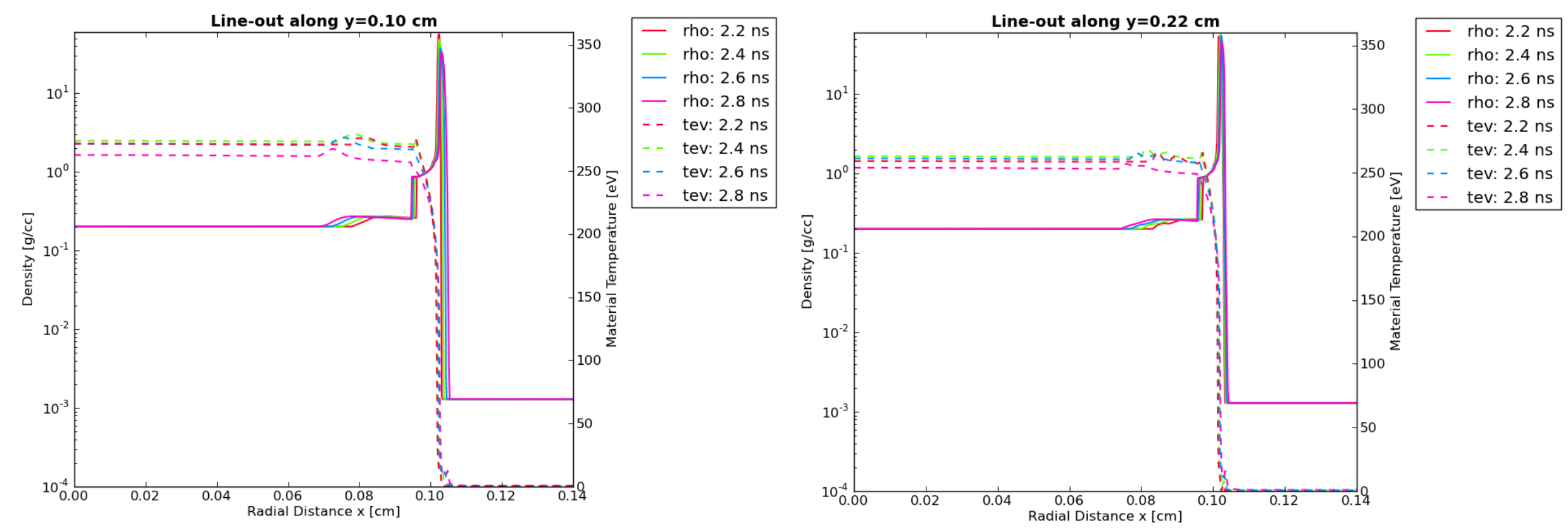

- Run 3D simulation with diagnostic slots in target

- Los Alamos

NATIONAL LABORATORY (ST, 1943

Operated by Los Alamos National Security, LLC for NNSA 


\section{Conclusions \& Future Work}

- How accurate are source models?

- Oxygen-doped target

- More detailed analysis of oxygen opacity over temperature range achievable

- Available diagnostics: back-lighter source, spectrometer

- Modify target and/or source incident on target to achieve conditions at BCZ?

- 3D simulation with diagnostic slots in target 


\section{Summary}

- This type of simulation useful for...

- But with limitations/caveats ... 


\section{Acknowledgements}

- Katie Mussack

- Paul Keiter

- Robert VanDervort

- Chris Orban

- Paul Drake 


\section{References}

1. P. A. Keiter et al., "Towards clarifying the solar abundance problem via opacity measurements at the National Ignition Facility," unpublished manuscript 2009.

2. J. E. Bailey, et al., "Experimental investigation of opacity models for stellar interior, inertial fusion, and high energy density plasmas," Physics of Plasmas 16, 058101 (2009).

3. A.S. Moore et al., "Developing high-temperature laser-driven half-hohlraums for the high-energydensity physics experiments at the National Ignition Facility," Fusion Science and Technology 63 (2013).

4. T.M. Guymer et al., "Quantifying equation-of-state and opacity errors using integrated supersonic diffusive radiation flow experiments on the National Ignition Facility," Physics of Plasmas 22, 043303 (2015).

5. C.A. Back et al., "Diffusive, supersonic $\mathrm{x}$-ray transport in radiatively heated foam cylinders," Physics of Plasmas 7, No. 5, (2000).

6. Jiang Shao-En et al., "Characteristic parameters of diffusive supersonic radiation transport in low density materials," Chinese Physics 16, No. 1 (2007).

7. C.L. Fryer et al., "Uncertainties in Radiation Flow Experiments," unpublished manuscript 2015. 


\section{Extra Slides}




\section{Gold Tube Ablation: 2.8 ns}

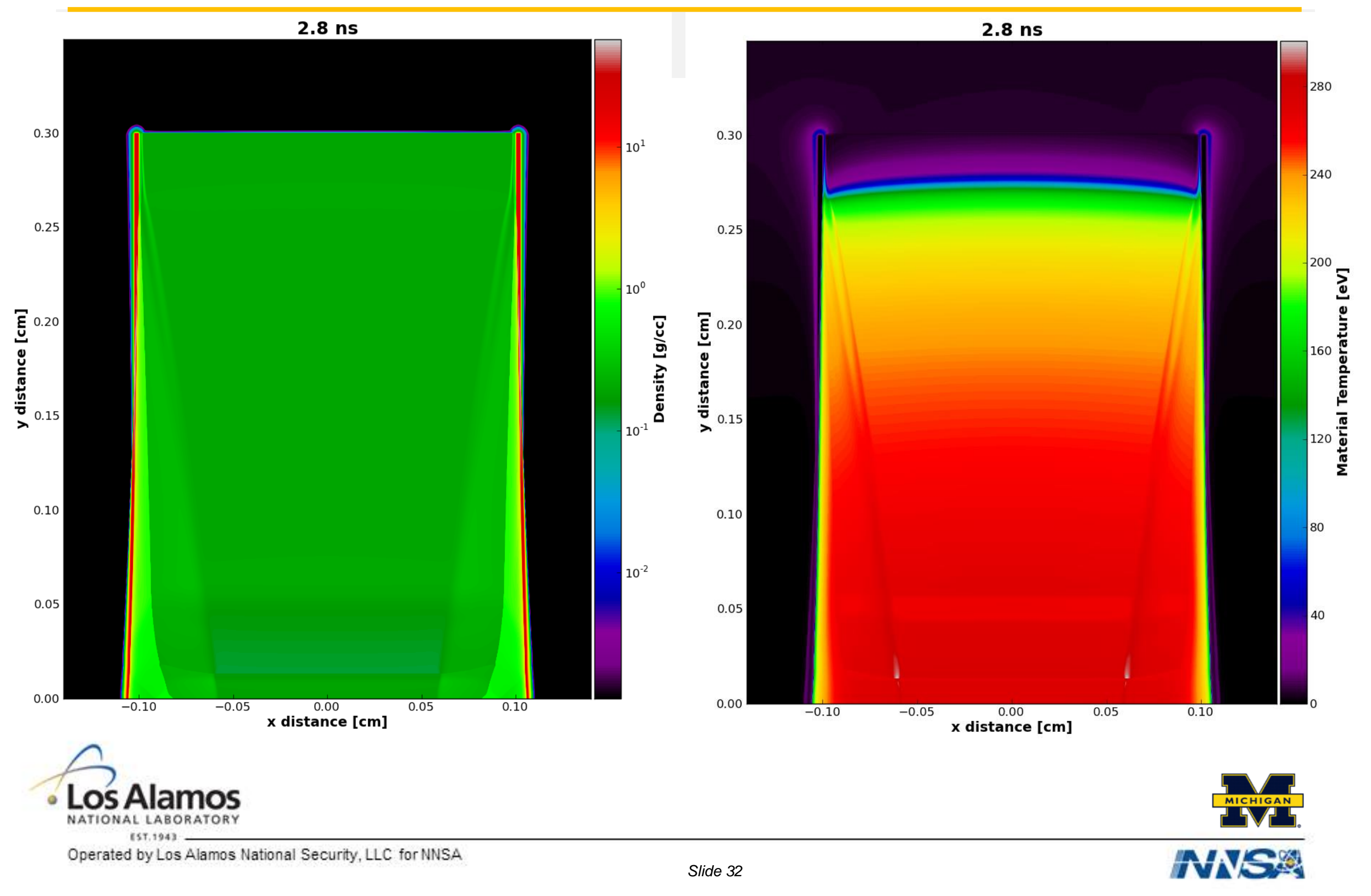




\section{Snd \& Prs @ 2.4 ns}
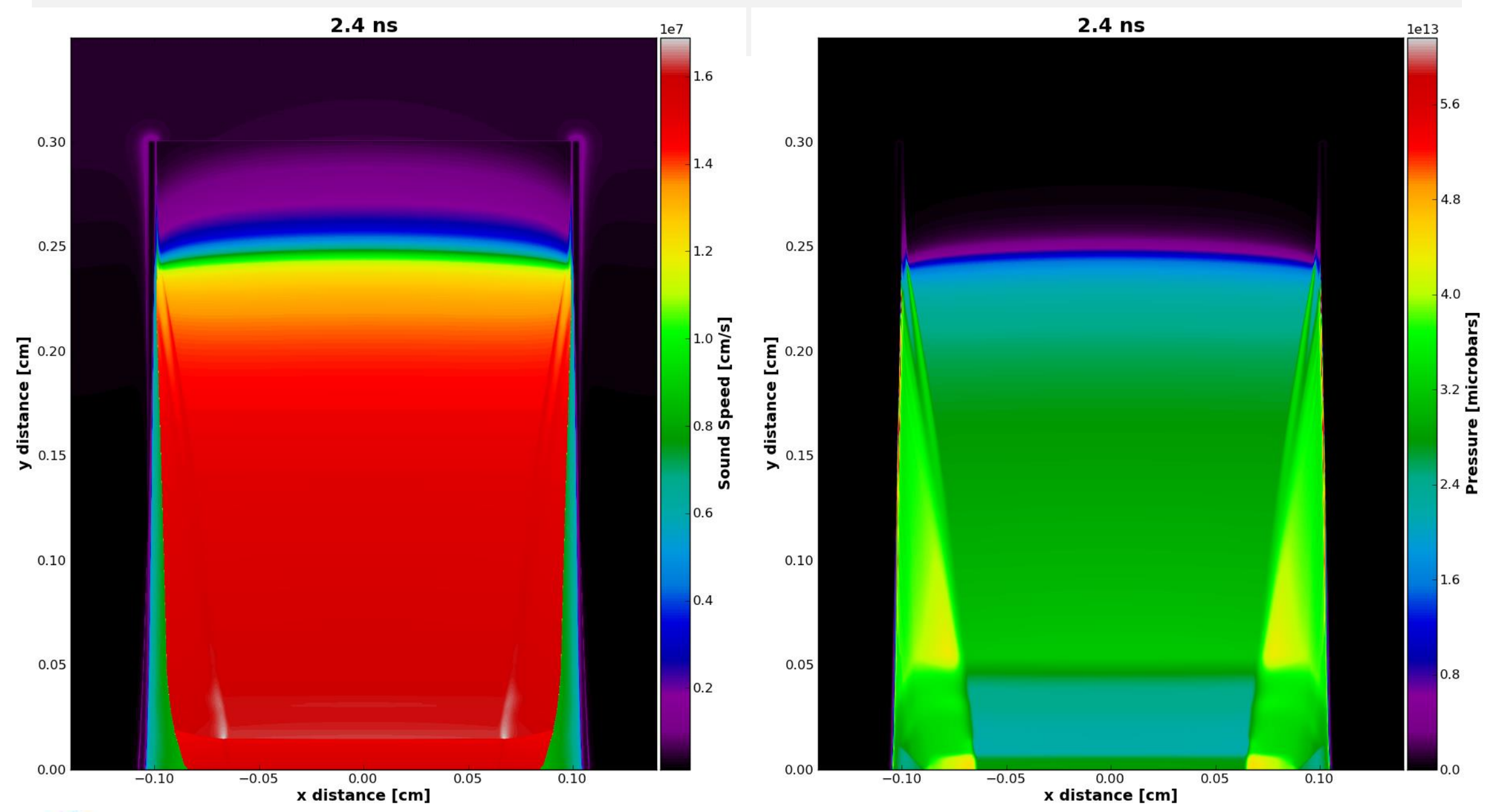

\section{- Los Alamos}

NATIONAL LABORATORY 


\section{Rev images for movie}

Use 'rev' images for $t=[0: 0.1: 6.5]$ ns to make movie
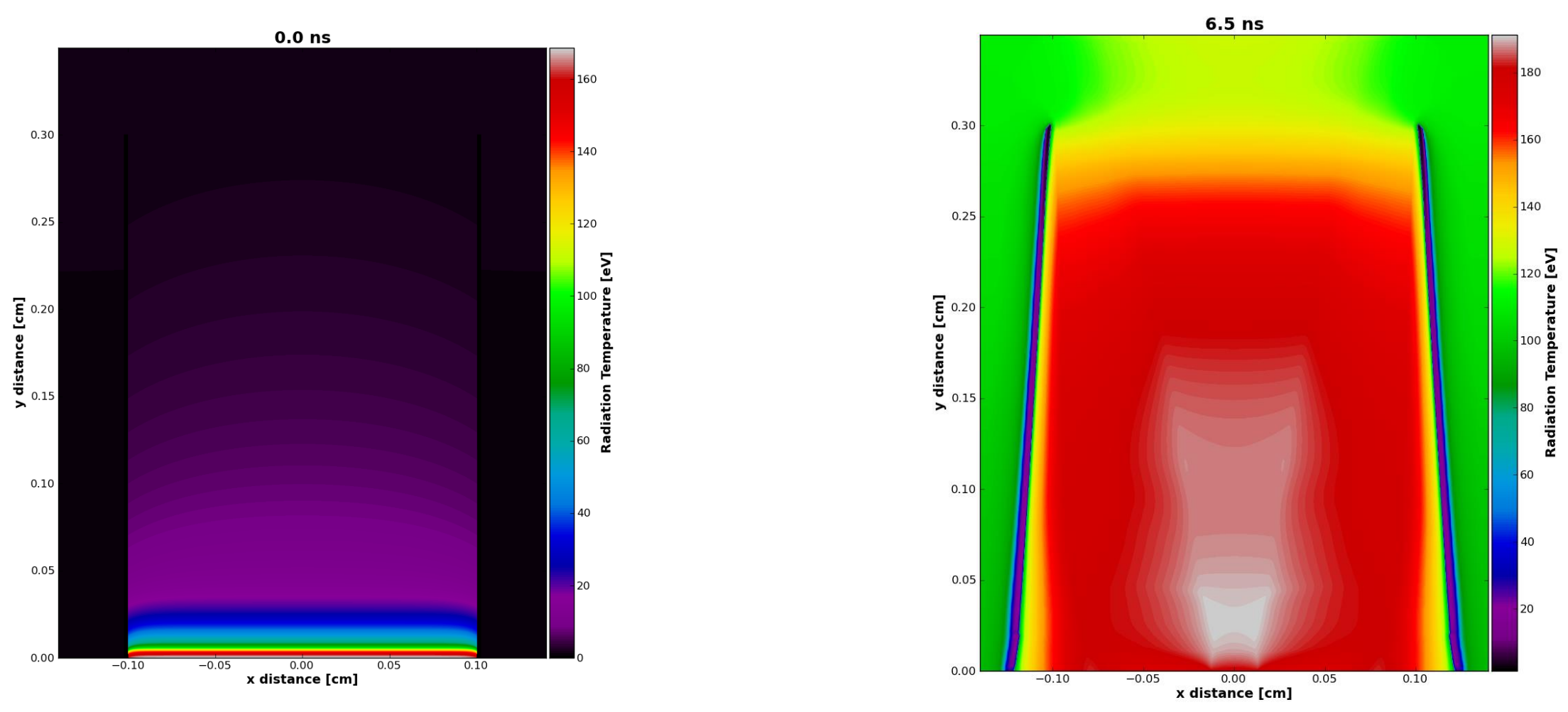

Los Alamos

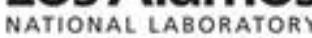




\section{Introduction}

We are developing a proposal for experiments at the National Ignition Facility (NIF) to measure opacities of elements with atomic number $Z$ in the range of 6-10 at temperatures and densities relevant to the base of the solar convection zone $(\sim 350 \mathrm{eV}, 0.2$ $\left.\mathrm{g} / \mathrm{cm}^{2}\right)$. Currently, there exist little da of measured opacities of materials within this high-energy-density (HED) regime. In astrophysics and ICF,

scientists must rely on opacity models to predict radiation transport behavio in HED plasmas. However, for $Z>2$ elements, opacity models do not agree. Experimentally measured opacity dat are necessary to validate opacity models or provide physical insights to inform the development of new opacity models.

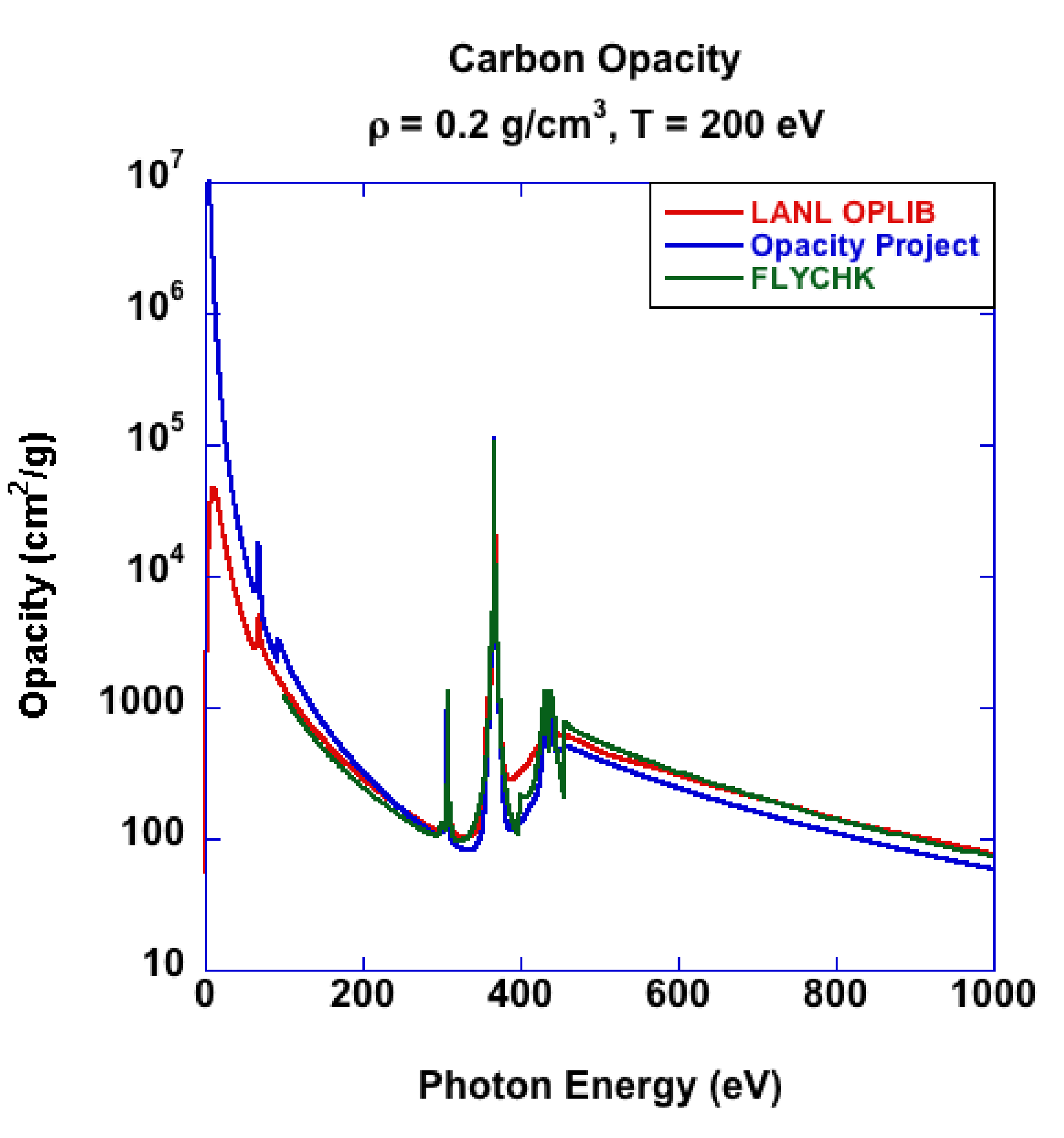

Fig. 1. Comparison of three widely used opacity models of carbon. Figure reproduced from [1]

\section{Previous experiments at $\mathrm{NIF}^{2}$ have demonstrated a high- temperature $(>340 \mathrm{eV}) \mathrm{X}$-ray radiation source with a Planckian distribution. Such a source is capable of generating a super- sonic radiation front within foan targets, a necessary condition for our opacity measurements. With sufficiently supersonic radiatio front, the density of the heated foam (behind the front) remain constant and equal to the density of the cold foam (ahead of the front) over a time scale of a couple nanoseconds. Afterwards, hydrodynamic effects create temperature and density gradients within the heated material, which would obfuscate analysis of opacity data. Therefore, there exists a brief time window during which the opacities of both the hot and cold material may be \\ measured at constant and well- \\ known densities.}

\section{xRage Simulation Results}

We are using xRage to simulate heat flow within our targets in order to estimate the me scale over which significant temperature and density gradients evolve. These imulations will better inform our target design and requirements for the spectral, spatial, and temporal resolution of the diagnostics to be used in the proposed experiments.

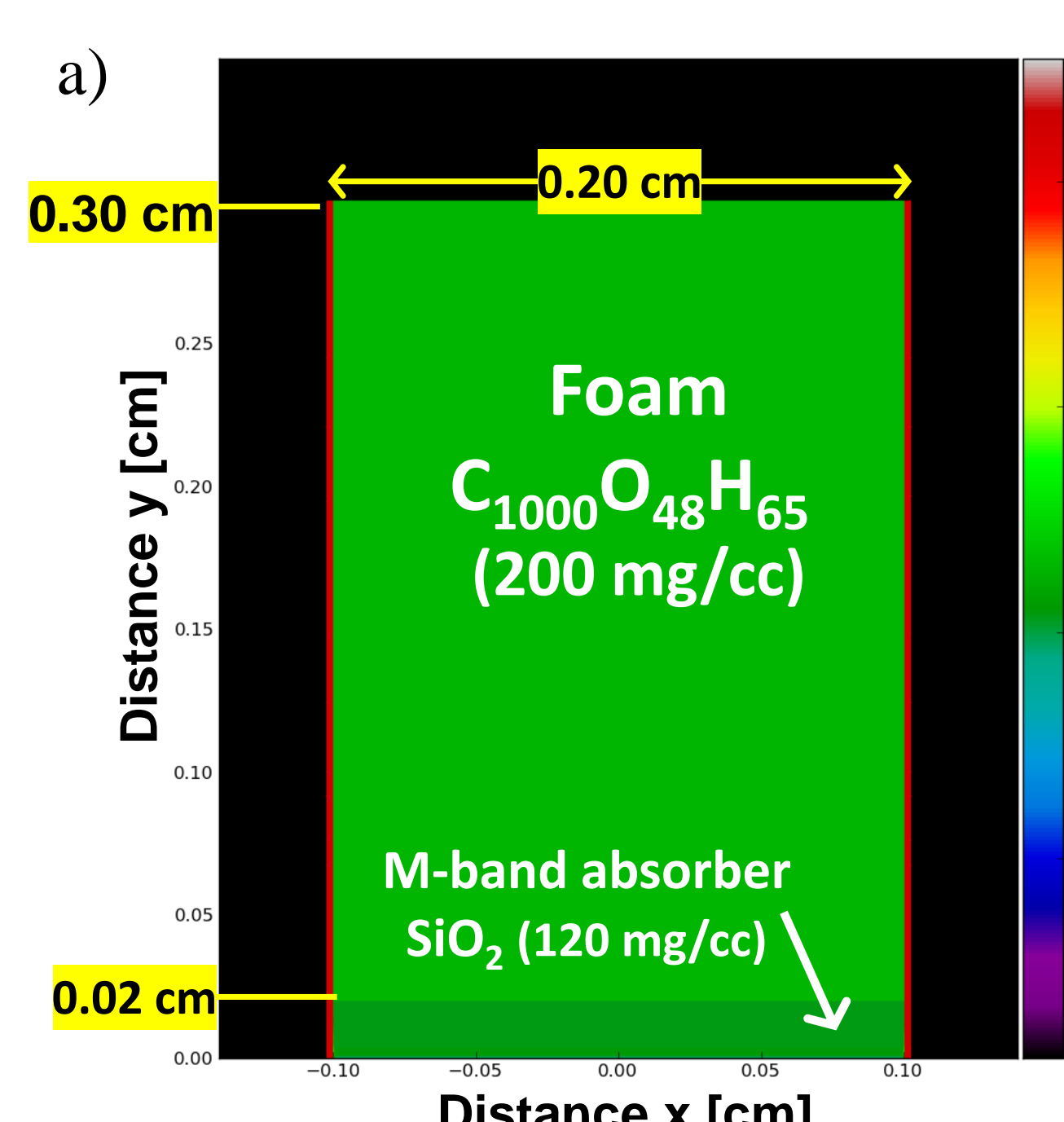

Distance $x[\mathrm{~cm}]$

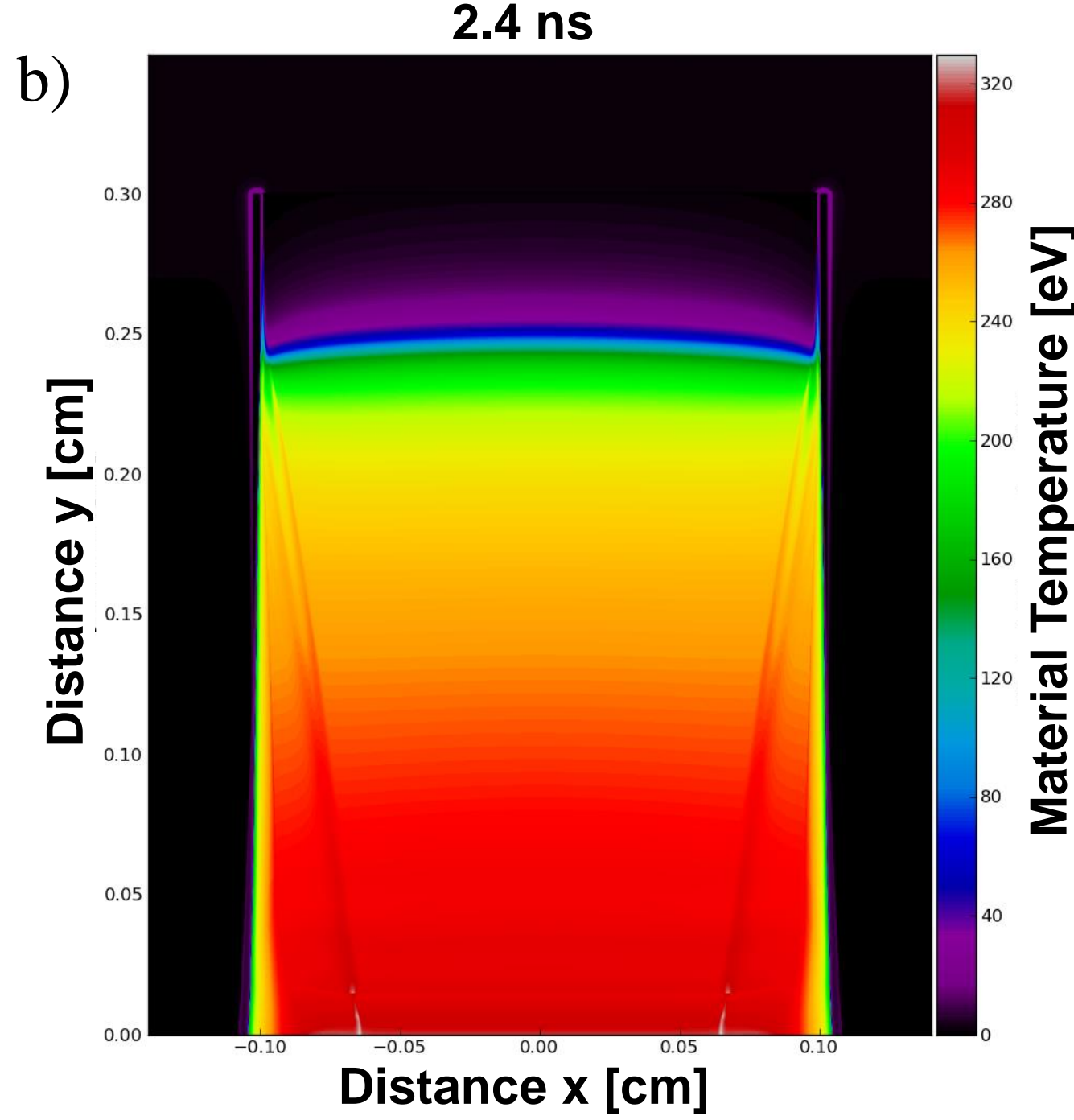

Distance $x[\mathrm{~cm}]$
Fig. 3. a) Target used in simulation. $\mathrm{A}$ thin $\mathrm{SiO}_{2}$ disk absorbs $\mathrm{M}$-band radiation emitted from the hohr aum. A gold tube with $25 \mu \mathrm{m}$ wall thickness surounds the for and ser

Simulation data collected includes material temperature (tev), radiation temperature (rev), density (rho), pressure (prs), and sound speed (snd). From this data, the radiation front location was defined as the point whe (she radiaton tenperature Mach number were then calculated.
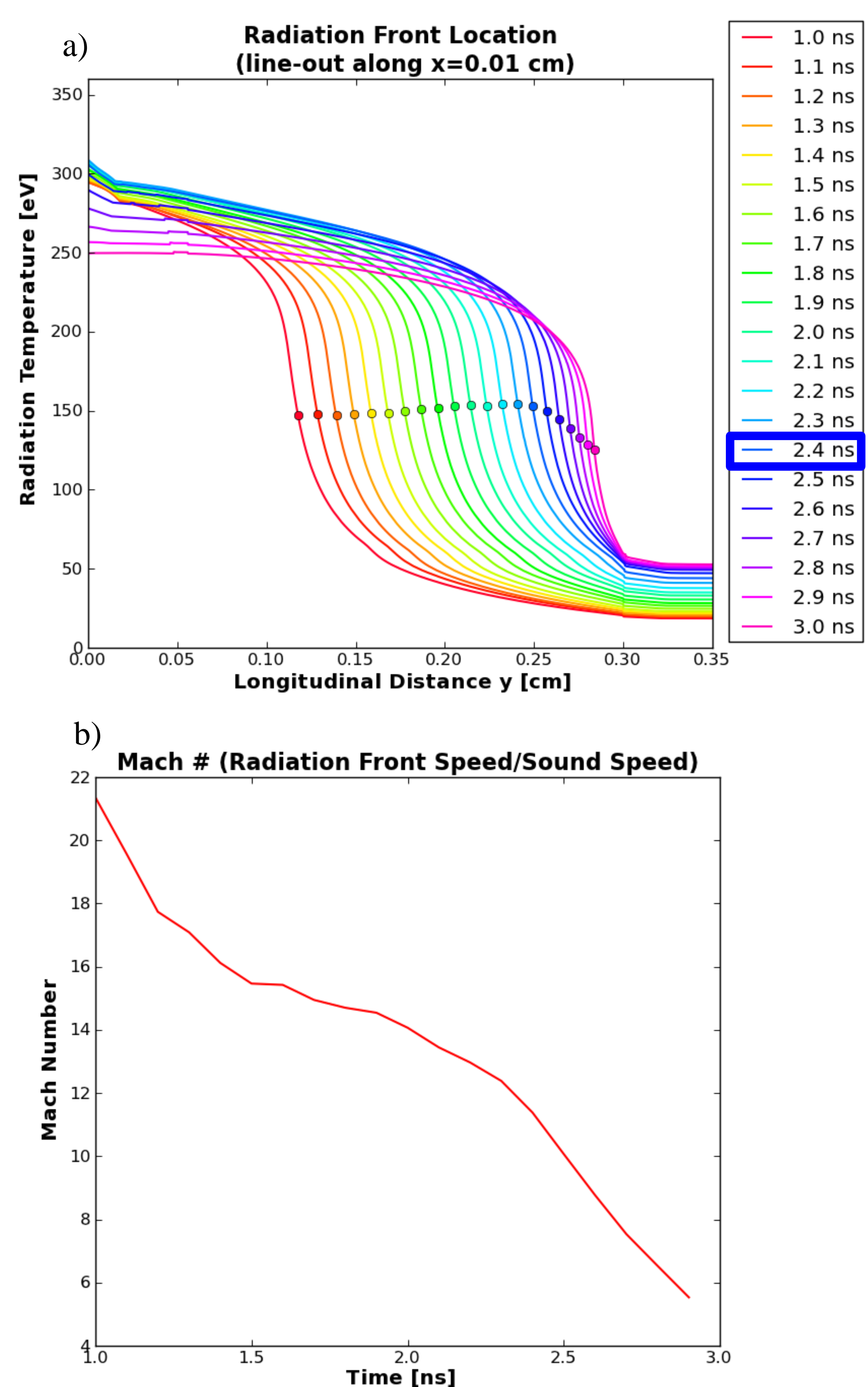

Fig. 4. a) Radiation temperature along center of target. Circles indicate location of radiation front used to calculate Mach number, shown in b). as a tamper. b) Material temperature in target $2.4 \mathrm{~ns}$ after radiation source is applied.

The heated foam reaches a maximum temperature $2.4 \mathrm{~ns}$ after the radiation source is applied. (This is consistent with the $2.5 \mathrm{~ns}$ laser pulse duration at NIF) At $2.4 \mathrm{~ns}$, the density of the heated foam is constant over a region $1.5 \mathrm{~mm}$ long (Figure 5a). The length of the target may be increased so that there is a similarly long region of constant-density cold foam. Looking at the cross section, density and temperature are uniform over a radial distance of $700 \mu \mathrm{m}$ at a distance $1.0 \mathrm{~mm}$ from the hot end of the target (Figure 5b).
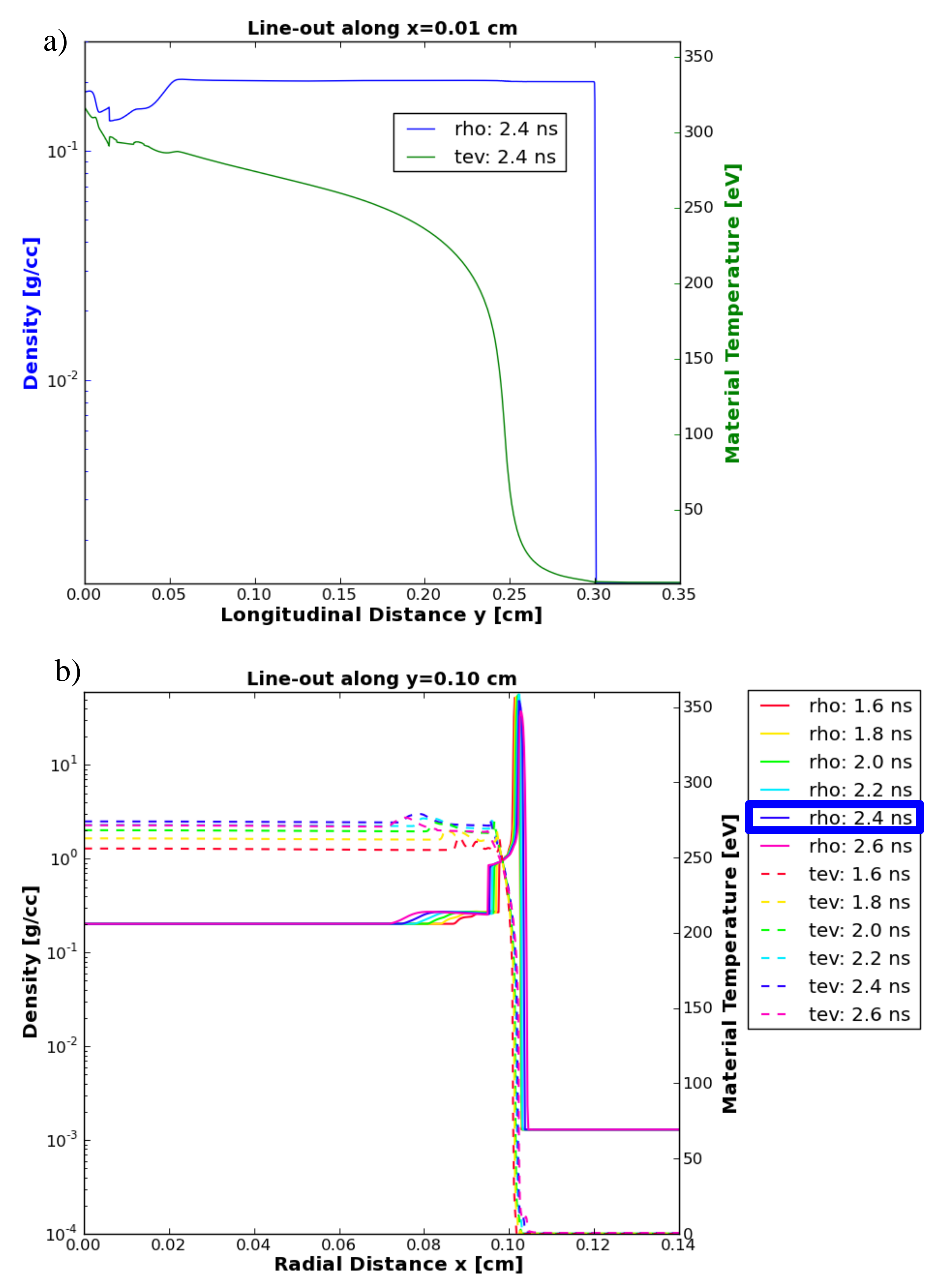

. an On-axis profiles of density and material temperature at 2.4 ns. b) Radial profiles of densily d material temperature for times $1.6-2.6 \mathrm{~ns}$ at a distance $1.0 \mathrm{~mm}$ from the hot end of the target.

\section{Conclusion \& Future Work}

Our simulations predict that the NIF laser source can drive a radiation front with Mach $>8$ over distances up to $2.5 \mathrm{~mm}$ within our foam targets. These conditions should enable the first opacity measurements of $\mathrm{C}, \mathrm{N}$ and $\mathrm{O}$ at temperatures and densities comparable to the base of the solar convection zone and yield valuable data for the refinement of opacity noders used mastophysics and ICF research. Next, we will consider the specifications of X-ray spectroneters and detectors avallable for the NIF experiments and design an imaging system for the target chamber. We must ensure that the sensitivity, spatial resolution and range of the diagnostics are sufficient for these opacity measurements. laser-driven half-hob-ray radiation source is emitted from a with the elements of interest (C, N, O) Opacities of these elements are found by measuring transmission spectra from X-ray backlighters through hot and cold regions of the foan targets.

\section{References \\ 1. P. Keiter $e$ t al., "Towards clarifying the solar abundance problem via opacity measurements at the National Ignition Facility." Unpublished manuscript, July 2015. 2. A.S. Moore et al., Fusion Science And Technology, 2013, Vol.63(2), pp.76-81.}

\section{Funding Acknowledgements}

U.S. Department of Energy, through the NNSA-DS and SC-OFES Joint Program in High-Energy-Density Laboratory Plasmas, grant number DE-NA0001840 